\title{
A combined stochastic programming and optimal control approach to personal finance and pensions
}

Konicz, Agnieszka Karolina; Pisinger, David; Rasmussen, Kourosh Marjani; Steffensen, Mogens

Published in:

OR Spectrum - Quantitative Approaches in Management

Link to article, DOI:

$10.1007 / \mathrm{s} 00291-014-0375-6$

Publication date:

2015

Link back to DTU Orbit

Citation $(A P A)$ :

Konicz, A. K., Pisinger, D., Rasmussen, K. M., \& Steffensen, M. (2015). A combined stochastic programming and optimal control approach to personal finance and pensions. OR Spectrum - Quantitative Approaches in Management, 37(3), 583-616. https://doi.org/10.1007/s00291-014-0375-6

\section{General rights}

Copyright and moral rights for the publications made accessible in the public portal are retained by the authors and/or other copyright owners and it is a condition of accessing publications that users recognise and abide by the legal requirements associated with these rights.

- Users may download and print one copy of any publication from the public portal for the purpose of private study or research.

- You may not further distribute the material or use it for any profit-making activity or commercial gain

- You may freely distribute the URL identifying the publication in the public portal 


\title{
A combined stochastic programming and optimal control approach to personal finance and pensions
}

\author{
Agnieszka Karolina Konicz* \\ agko@dtu.dk
}

Kourosh Marjani Rasmussen

kmra@dtu.dk

\author{
David Pisinger \\ dapi@dtu.dk
}

\author{
Mogens Steffensen \\ mogens@math.ku.dk
}

April 30, 2014

\begin{abstract}
We combine a dynamic programming approach (stochastic optimal control) with a multi-stage stochastic programming approach (MSP) in order to solve various problems in personal finance and pensions. Stochastic optimal control produces an optimal policy that is easy to understand and implement. However, explicit solution may not exist, especially when we want to deal with constraints, such as limits on portfolio composition, limits on the sum insured, an inclusion of transaction costs or taxes on capital gains, which are important issues regularly mentioned in the literature. Both optimization methods are integrated into one MSP formulation, and in a short computational time produce a solution, which takes into account the entire lifetime of an individual with a focus on the practical constraints during the first years of a contract.

Two applications are considered: (A) optimal investment, consumption and sum insured for an individual maximizing the expected utility of consumption and bequest, and (B) optimal investment for a pension saver who wishes to maximize the expected utility of retirement benefits. Numerical results show that among the considered practical constraints, the presence of taxes affects the optimal controls the most. Furthermore, the individual's preferences, such as impatience level and risk aversion, have even a higher impact on the controlled processes than the taxes on capital gains.
\end{abstract}

\section{Introduction}

The purpose of this paper is to formulate and solve two optimization problems relevant for personal finance and pensions in a multi-period stochastic framework. Problem (A) investigates the optimal investment, consumption and sum insured for an individual maximizing the utility of consumption and a terminal utility of leaving a positive amount of money upon death over an uncertain lifetime. Problem (B) is relevant for a pension product design in a defined contribution plan. A pension saver wishes to maximize the utility of the future retirement benefits by optimally controlling the investment both before and after retirement as well as the level of the benefits that will be received after

\footnotetext{
${ }^{*}$ Corresponding author at: DTU Management Engineering, Management Science, Technical University of Denmark, Produktionstorvet 426, 2800 Kgs. Lyngby, Denmark. Tel.: +45 4525 3109; Fax: +45 4525 3435; email: agko@dtu.dk
} 
retirement. The solution determines a pension product that reflects the individual's risk preferences and impatience level.

A classical approach for consumption-investment optimization problems is stochastic optimal control, also referred to continuous time and state dependent dynamic programming. Stochastic optimal control is common in financial and actuarial literature and focuses on deriving the explicit (analytical) solutions to a given model. Probably the most influential papers in this field have been written by Merton (1969, 1971) who defined the original problem of optimizing utility of consumption and terminal wealth over a fixed time horizon for an investor. This work has inspired many researchers who either expanded the original model or investigated different objective functions, by introducing for instance the lifetime uncertainty, sum insured and the labor income, Richard (1975), stochastic interest rate, Munk and Sørensen (2004), salary uncertainty, Cairns et al. (2006), multiperson household, Bruhn and Steffensen (2011), borrowing constraints, Byung and Yong (2011), or constant linear taxation, Bruhn (2013). Applications within defined contribution pension scheme with a focus on the investment strategy either during the accumulation or post retirement phase (decumulation) together with the optimal time of annuitization, have been considered by Milevsky and Young (2007) and Gerrard et al. (2004, 2012).

The main advantage of a stochastic optimal control approach is the analytical form of the optimal solution, which is easy to understand and implement. However, the main drawback of this approach is that the explicit solution in many cases does not exist, especially, when dealing with more realistic constraints, such as limits on portfolio composition, limits on the sum insured, an inclusion of transaction costs or taxes on capital gains, which are important issues regularly mentioned in the literature. Therefore, to overcome these limitations, researchers must apply numerical methods. To focus on the practicalities of the problem, we choose in this paper a multi-stage stochastic programming (MSP) approach, which is broadly applied in operations research. MSP is a general purpose framework for modelling optimization problems where an objective function can take a variety of forms. It is based on the scenario trees that represent the range of possible outcomes for the uncertainties. Rather than finding a generic optimal policy, the optimal solution is computed numerically at each node in the scenario tree, for the specified decision variables. MSP can easily address realistic considerations and constraints, as long as they have an algebraic form.

A stochastic programming approach is more common within portfolio management and assetliability management than within the areas concerning the individual investors such as optimal investment-consumption decisions. See e.g. Mulvey et al. (2006, 2007) who argue that multi-period investment models combined with Monte Carlo simulation can address important considerations for long-term investors, and Ferstl and Weissensteiner (2010) who present a stochastic linear programming model for optimal asset allocation in a situation where a financial company wishes to minimize the conditional value at risk. Nevertheless, applying stochastic programming or other numerical approaches to find the optimal decisions from the individual investors' point of view can also be found in the literature. See e.g. Horneff et al. (2008) considering an individual characterized by Epstein-Zin 
preferences, Cai and Ge (2012) investigating the asset allocation for an investor with a loss aversion objective, a predetermined objective and a greedy objective, Consigli et al. (2012) comparing investment opportunities offered by traditional pension products and unit-linked contracts with variable life annuities, and Blake et al. (2013) deriving the optimal investment for a loss averse pension saver with an interim and final target.

However, the main drawback of a multi-stage stochastic programming approach is that the problem size grows quickly as a function of the number of periods and scenarios. In particular, taking into consideration the entire lifetime of an individual can be challenging in terms of computational tractability. Several studies investigated the methods that could overcome this issue. For example, Grinold (1977, 1983) presents and compares different methods of approximating the general multistage optimization problem upon horizon, and concludes that a dual equilibrium technique will give improving approximations of the optimal solution as the horizon increases, and perfect approximations in the limit. Barro and Canestrelli 2005, 2006) show that a dynamic portfolio problem written as a multistage stochastic program can be rewritten as a discrete time optimal control problem, whereas Barro and Canestrelli (2011) further extends this work to a broader class of multistage stochastic programming problems, and reformulates the problems as discrete time stochastic optimal control problems. Due to this reformulation one can solve large optimization problems in a low computational time.

Finally, Geyer et al. (2009a) argue that stochastic optimal control and stochastic programming can be combined and integrated into one multi-stage programming formulation. The authors model the optimal investment-consumption problem using stochastic linear programming and combine it with the closed-form solution obtained by Richard (1975). The mixed approach can accurately replicate the first-stage investment and consumption decisions derived by Richard (1975). They argue that stochastic programming and stochastic optimal control complement each other, especially in the areas where one or the other does not perform well on its own. Thus, the main advantage of the mixed approach is the optimal solution that is obtained under the realistic assumptions and within a short computational time.

Inspired by the advantages of the mixed approach, we apply this method for two optimization problems relevant for personal finance and pensions. Problem (A), similarly to Geyer et al. (2009a), is based on the explicit solution derived by Richard (1975). Our paper, however, differs from the model presented in Geyer et al. (2009a) in several aspects. The most important improvement is adding the sum insured to the model. The explicit solutions for optimal consumption, investment and sum insured derived by Richard (1975) assume the life insurance policy in the model. Therefore, the sum insured must be included in the MSP formulation. Furthermore, we focus on the optimal controls at all stages, and not only the first-stage decision as done in Geyer et al. (2009a). Finally, instead of approximating the objective function by a piecewise-linear interpolant, we solve the problem directly using a nonlinear solver, thus removing the approximation error from the results.

Our work differs from Richard (1975) by including realistic constraints such as limits on portfolio 
composition, limits on the sum insured, an inclusion of transaction costs and taxes on capital gains. We show that the optimal investment and sum insured derived by Richard (1975) is for a large variety of parameters problematic from a practical point of view. First, the optimal investment implies shorting the risk-free asset and gearing the mutual fund, which is often a limitation for a private investor. Second, the sum insured is for most of the individual's lifetime negative, which cannot happen in practice. Third, while transaction costs have a minor impact on the optimal controls, the presence of taxes on capital gains affects the optimal investment strategy significantly.

Another contribution of this paper is a formulation and the solution for problem (B). This problem is relevant for pension product design in defined contribution pension plans, and its uniqueness from the classical stochastic optimal control perspective lies in defining the optimal investment for the period before and after retirement, whereas the optimal consumption only during the period after retirement. All the aforementioned studies investigating the optimal investment-consumption problems consider the optimal controls over the same period; none of them defines the optimal controls over different periods. Therefore, we derive the explicit formula for the optimal value function and the optimal controls using Hamilton-Jacobi-Bellman techniques for such a model. We further solve problem (B) by using the mixed optimization approach.

The paper is organized as follows. Section 2 describes problems (A) and (B) in more detail together with their optimal solutions obtained via dynamic programming. Section 3 explains how to build a bridge between dynamic programming and multi-stage stochastic programming by incorporating both optimization methods in one MSP formulation. Section 4 focuses on defining the MSP model, i.e. the objective function and the constraints, and scenario tree generation. Section 5 presents the numerical results of the optimal controls obtained via two different optimization methods, and the impact on the controls of the modifications considered during the first years of the contract. Finally, Section 6 summarizes the paper and suggests the future work. The paper includes two appendices: Appendix A explains the application of the mutual fund theorem, and Appendix B presents a derivation of the optimal value function and controls for problem (B).

\section{Model description}

The section presents the general model assumptions relevant for problems (A) and (B).

One of the main decisions for the individual/pension fund is how to allocate the savings between different financial assets. We assume that the savings can be invested in $N$ assets: one risk-free (bonds) and $N-1$ risky assets (stocks). Given that the economy is represented by a standard Brownian motion $W$ defined on the measurable space $(\Omega, \mathcal{F})$, where $\mathcal{F}$ is the natural filtration of $W$, 
the asset prices $S_{t}^{i}$ can be modeled by a geometric Brownian motion,

$$
\begin{aligned}
d S_{t}^{i} & =\alpha_{i} S_{t}^{i} d t+\sigma_{i} S_{t}^{i} d W_{t}^{i} \\
S_{0}^{i} & =s_{0}^{i}>0
\end{aligned}
$$

where $\alpha_{i}$ and $\sigma_{i}$ are constants, and $\forall_{\{i, j\}=1, \ldots, N-1} d W_{t}^{i}$ and $d W_{t}^{j}$ are correlated with a coefficient $\operatorname{corr}_{i j}$. The risk-free asset is defined by $\alpha_{N}=r$ and $\sigma_{N}=0$. Merton (1971) shows that without loss of generality we can assume that all the risky assets are included in one mutual fund, the prices of which are modeled by a geometric Brownian motion,

$d S_{t}=\alpha S_{t} d t+\sigma S_{t} d W_{t}$

where $\alpha$ is the expected rate of return on the risky fund and $\sigma$ is the volatility of the risky fund. See App. A for details. We also assume that the individual is allowed to borrow money at the risk-free rate $r$ in order to buy the risky assets, as well as to take a short position in those assets.

Further, assume that $\mathbb{P}$ and $\mathbb{P}^{*}$ are equivalent probability measures on the measurable space $(\Omega, \mathcal{F})$. $\mathbb{P}$ denotes the objective measure, whereas $\mathbb{P}^{*}$ is used by the pension fund for pricing both market and life insurance risk. Thus, consistently with Richard (1975)'s assumptions, we consider life insurance policies as standard tradable financial contracts 1

The individual has an uncertain lifetime modeled by a finite state Markov chain $Z$, defined on a measurable space $(\Omega, \mathcal{F})$. The state process $Z$ indicates whether or not the person is alive; it takes values in $\{0,1\}$, and starts in 0 at time 0 , i.e. the person is alive. The mortality rates, $\mu_{t}$ and $\mu_{t}^{*}$ (respectively, under $\mathbb{P}$ and $\mathbb{P}^{*}$ ) are defined by the jump intensities of the process $Z$. They are assumed to be continuous and deterministic (as defined later in Sec. 5) and satisfy $\mu_{t} \rightarrow \infty$ and $\mu_{t}^{*} \rightarrow \infty$, which implies that $\lim _{t \rightarrow \infty} \mathbb{P}\left(Z_{t}=1\right)=\lim _{t \rightarrow \infty} \mathbb{P}^{*}\left(Z_{t}=1\right)=1$.

The cash-flows accompanying the savings account are formalized by the continuous processes: a deterministic contribution process $l_{t}$ that, depending on the problem, is interpreted either as the labor income that is contributed to the account or the percentage of the labor income that is paid to the retirement savings (premiums) ${ }^{2}$ and a consumption process $c_{t}$ determining the consumption of the savings for the private purposes or the benefits that the pension fund pays to the retiree.

Finally, we assume that the individual is risk averse and has a CRRA utility function $u$ characterized by a constant relative risk aversion $1-\gamma$, constant elasticity of intertemporal substitution $1 /(1-\gamma)($ EIS $)$ and time dependent weights $w_{t}$ reflecting the importance of present consumption in

\footnotetext{
${ }^{1} \mathrm{~A}$ similar approach to life insurance contracts can also be found in Kraft and Steffensen (2008). The assumption about tradability of life insurance is not substantially different from considering a case where policy holders are allowed to make alterations to their contracts. Apart from realistic issues with health and other types of assymetric information (which do not appear in our model), this is certainly what appears in practice.

${ }^{2}$ The model could be extended by adding a stochastic contribution process. However, the explicit solutions to problems (A) and (B) can be derived only if the labor income is assumed to be spanned by the stock risk. Otherwise, the explicit solutions to the control problems do not exist.
} 
contrast to future consumption characterized by the impatience factor $\rho$ :

$u(t, c)=\frac{1}{\gamma} w_{t}^{1-\gamma} c^{\gamma}, \quad$ where $\quad w_{t}^{1-\gamma}=e^{-\rho t}$.

Parameter $\gamma$ is defined for $(-\infty, 1) \backslash\{0\}$, whereas for $\gamma=0$ we have the case of the logarithmic utility.

Below, we define the model setup specific for the particular problems.

\subsection{Problem (A) - optimal investment, consumption and sum insured}

We keep the original settings defined in Richard (1975) and recall only the most important assumptions and results that are crucial for this paper.

Savings dynamics. The individual has a bequest motive and upon death her heirs receive an amount on the savings account plus the sum insured, $X_{t}+I_{t}$. The premium for the coverage is $\mu_{t}^{*} I_{t}$, where $\mu_{t}^{*}$ is the natural premium intensity decided by the life insurance company, also called pricing mortality. In particular, we allow for negative $I_{t}$, which means that the person sells the amount $I_{t}$ to the pension fund and purchases life annuities.

The wealth dynamics while the person is alive develop as follows:

$$
\begin{aligned}
d X_{t} & =\left(r+\pi_{t}(\alpha-r)\right) X_{t} d t+\pi_{t} \sigma X_{t} d W_{t}+l_{t} d t-c_{t} d t-\mu_{t}^{*} I_{t} d t \\
X_{0} & =x_{0}
\end{aligned}
$$

where $r$ is the return on the risk-free asset, $\alpha$ is the expected rate of return on the mutual fund, $\sigma$ is the volatility of the mutual fund, $\pi$ is the proportion invested in the mutual fund, and $1-\pi$ in the risk-free asset.

Optimization problem. Since the individual has a bequest motive, she obtains the utility from the amount that she will leave upon death to her heirs:

$U(t, x)=\frac{1}{\gamma} v_{t}^{1-\gamma} x^{\gamma}$

where $v$ is the weight for this utility. It sounds reasonable to define $v$ in terms of the weights for the utility of consumption $w$, hence $v$ denotes the weight put on her heir's consumption relative to her own,

$v_{t}^{1-\gamma}=\lambda^{-\gamma} w_{t}^{1-\gamma}$

where $\lambda$ is a constant. The individual can control consumption, investment and sum insured, in order to maximize the utility of consumption and bequest. Given the wealth dynamics, $X_{t}$, the problem 
is mathematically formulated as:

$$
\begin{aligned}
V^{A}(t, x) & =\sup _{\pi, c, I \in \mathcal{Q}[t, \widetilde{T})} E_{t, x}\left[\int_{t}^{\widetilde{T}} e^{-\int_{t}^{s} \mu_{\tau} d \tau}\left(u\left(s, c_{s}\right)+\mu_{s} U\left(s, X_{s}+I_{s}\right)\right) d s\right], \\
V^{A}(\widetilde{T}, x) & =0
\end{aligned}
$$

where $E_{t, x}$ is the conditional expectation under $\mathbb{P}$, given that the person is alive at time $t$ and holds wealth $X_{t}=x$, and $\mathcal{Q}[t, \widetilde{T})$ is the set of control processes for the time $[t, \widetilde{T})$ which are admissible

at time $t . \widetilde{T}$ is a fixed time point at which the investor is dead with certainty. Both utilities are multiplied by factor $e^{-\int_{t}^{s} \mu_{\tau} d \tau}$ denoting the probability that the individual survives until time $s>t$, given she has survived until time $t$. Furthermore, the utility of bequest is multiplied by the mortality intensity rate $\mu_{s}$, which represents the probability that the person dies within a short period after time $s$. The utility functions $u$ and $U$ are assumed to be strictly concave in $c$ and $X+I$, respectively.

The optimal value function for this problem is given by

$V^{A}(t, x)=\frac{1}{\gamma} f_{A}(t)^{1-\gamma}\left(x+g_{A}(t)\right)^{\gamma}$

where

$$
\begin{aligned}
& f_{A}(t)=\int_{t}^{\widetilde{T}} e^{-\frac{1}{1-\gamma} \int_{t}^{s}\left(\mu_{\tau}-\gamma\left(\mu_{\tau}^{*}+\varphi\right)\right) d \tau}\left[w_{s}+\left(\frac{\mu_{s}}{\left(\mu_{s}^{*}\right)^{\gamma}}\right)^{1 /(1-\gamma)} v_{s}\right] d s, \quad \varphi=r+\frac{(\alpha-r)^{2}}{2 \sigma^{2}(1-\gamma)}, \\
& g_{A}(t)=\int_{t}^{\widetilde{T}} e^{-\int_{t}^{s}\left(r+\mu_{\tau}^{*}\right) d \tau} l_{s} d s
\end{aligned}
$$

and the optimal investment, consumption and sum insured are of the form:

$$
\pi_{t}^{*}=\frac{\alpha-r}{\sigma^{2}(1-\gamma)} \frac{X_{t}+g_{A}(t)}{X_{t}}, \quad c_{t}^{*}=\frac{w_{t}}{f_{A}(t)}\left(X_{t}+g_{A}(t)\right), \quad I_{t}^{*}=\left(\frac{\mu_{t}}{\mu_{t}^{*}}\right)^{1 /(1-\gamma)} \frac{v_{t}}{f_{A}(t)}\left(X_{t}+g_{A}(t)\right)-X_{t} .
$$

Equations (4A - (7A) correspond to equations (25) and (40)-(44) in Richard (1975).

\subsection{Problem (B) - optimal investment with optimal life annuities}

The application of this problem is relevant for a pension product design in a defined contribution pension scheme, both occupational and private. As seen in most of the European pension markets, premiums are defined as a fixed percentage of the salary. In the occupational pension schemes this percentage is typically decided by the employer, whereas private schemes allow the customer to choose both the size and the frequency of the premiums. When entering the contract, the individual is often given a choice of different pension products characterized by various investment strategies and a type of benefits. For example, one can choose between a conservative, moderate or aggressive 
investment strategy, but it is the pension fund that decides how to invest the savings such that it reflects the customer's preferences. In a different kind of product, such as a unit-linked, it is the pension saver who can decide on the portfolio allocation and adjust it during the contract as she wishes. Both types of products typically offer an option to add a guarantee to the contract, but in this work we assume that the benefits are directly linked to the market and no guarantees are provided. In terms of the benefits, one can typically choose between a lump sum payment $(\widetilde{T}=T)$, life annuities $(\widetilde{T} \rightarrow \infty)$ or temporary life annuities $(10-25$ years, $\widetilde{T}<\infty)$. The size of the benefits is calculated by the pension fund according to the actuarially fair principles.

The considered optimization problem allows for controlling the investment strategy both before and after retirement, as well as the size and distribution of the benefits after retirement. The optimal solution determines a product that is customized to the individual's risk and impatience preferences. In case of death, the pension fund inherits all the individual's savings.

Savings dynamics. We split the problem into a before retirement period (accumulation phase, $t<T$ ) and an after retirement period (decumulation phase, $t \geq T$ ). In practice the pension saver is not allowed to withdraw the pension savings for the consumption reasons or receive the benefits before retirement, and it is also reasonable to assume that the premiums paid to the pension fund are set to 0 after retirement. Thus, $c_{t}=0$ for $t \in\left[t_{0}, T\right)$ and $l_{t}=0$ for $t \in[T, \widetilde{T})$. Since the person has no bequest motive, she has an additional income of $\mu_{t}^{*} X_{t}$, which is the amount that the pension fund pays to be her only inheritor. With these assumptions the definition of savings dynamics are of the form

$$
\begin{aligned}
d X_{t} & = \begin{cases}\left(r+\pi_{t}(\alpha-r)+\mu_{t}^{*}\right) X_{t} d t+\pi_{t} \sigma X_{t} d W_{t}+l_{t} d t, & t \in\left[t_{0}, T\right), \\
\left(r+\pi_{t}(\alpha-r)+\mu_{t}^{*}\right) X_{t} d t+\pi_{t} \sigma X_{t} d W_{t}-c_{t} d t, & t \in[T, \widetilde{T}),\end{cases} \\
X_{t} & =x_{0} .
\end{aligned}
$$

Optimization problem. The pension saver wishes to maximize the utility of pension benefits, or in other words, the utility of consumption after retirement, while being able to control the investment strategy and the distribution of the benefits. She obtains no utility from consumption before retirement, i.e. $u(t, c)=0$ for $t \in\left[t_{0}, T\right)$, hence the problem is formulated as follows:

$$
\begin{aligned}
V^{B}(t, x) & =\sup _{\pi \in \mathcal{Q}[t, \widetilde{T}), c \in \mathcal{Q}[\max (t, T), \widetilde{T})} E_{t, x}\left[\int_{\max (t, T)}^{\widetilde{T}} e^{-\int_{t}^{s} \mu_{\tau} d \tau} u\left(s, c_{s}\right) d s\right], \\
V^{B}(\widetilde{T}, x) & =0 .
\end{aligned}
$$

where, as before, $E_{t, x}$ is the conditional expectation under $\mathbb{P}$, given that the person is alive at time $t$ and holds savings $X_{t}=x$, and $\mathcal{Q}[t, \widetilde{T})$ is the set of control processes for time $[t, \widetilde{T})$ which are admissible at time $t$. The control processes in this problem are the proportion in the risky fund 
$\pi_{t}$ and the benefits $c_{t}$. As in problem (A), we take into consideration the uncertain lifetime of the individual by multiplying the utility function by the factor $e^{-\int_{t}^{s} \mu_{\tau} d \tau}$. At time $\widetilde{T}$ the investor is assumed to be dead with certainty. The utility function $u$ is assumed to be strictly concave in $c$.

Even though the individual obtains no utility from consumption before retirement, the investment process $\pi_{t}$ is controlled both before and after retirement. Thus, the novelty of this problem from the stochastic optimal control point of view lies in defining the optimal consumption and investment over different (but partially overlapping) periods. The research to date has tended to focus on deriving the explicit solution for the controls over the same period; either until retirement, see e.g. Cairns et al. (2006) and Bruhn and Steffensen (2011), after retirement, see e.g. Gerrard et al. (2004, 2006) and He and Liang (2013), or generally over the life cycle, see e.g. Merton (1969, 1971), Richard (1975), Milevsky and Young (2007), and Kraft and Steffensen (2008). Because, to the best of our knowledge, neither Problem (B) or the case of deriving optimal controls over different periods has been considered by other researchers, we derive the optimal value function and the optimal controls, see Appendix B for details.

The optimal value function is of the form:

$V^{B}(t, x)=\frac{1}{\gamma} f_{B}(t)^{1-\gamma}\left(x+g_{B}(t)\right)^{\gamma}$,

where

$$
\begin{aligned}
& \begin{cases}f_{B}(t)=e^{-\frac{1}{1-\gamma} \int_{t}^{T}\left(\mu_{\tau}-\gamma\left(\mu_{\tau}^{*}+\varphi\right)\right) d \tau} \int_{T}^{\widetilde{T}} e^{-\frac{1}{1-\gamma} \int_{T}^{s}\left(\mu_{\tau}-\gamma\left(\mu_{\tau}^{*}+\varphi\right)\right) d \tau} w_{s} d s, & t \in\left[t_{0}, T\right), \\
f_{B}(t)=\int_{t}^{\widetilde{T}} e^{-\frac{1}{1-\gamma} \int_{t}^{s}\left(\mu_{\tau}-\gamma\left(\mu_{\tau}^{*}+\varphi\right)\right) d \tau} w_{s} d s, & t \in[T, \widetilde{T}),\end{cases} \\
& \begin{cases}g_{B}(t)=\int_{t}^{T} e^{-\int_{t}^{s}\left(r+\mu_{\tau}^{*}\right) d \tau} l_{s} d s, & t \in\left[t_{0}, T\right), \\
g_{B}(t)=0, & t \in[T, \widetilde{T}),\end{cases}
\end{aligned}
$$

and the optimal controls are given by

$\pi_{t}^{*}=\frac{\alpha-r}{\sigma^{2}(1-\gamma)} \frac{X_{t}+g_{B}(t)}{X_{t}}, \quad t \in[t, \widetilde{T}), \quad c_{t}^{*}=\frac{w_{t}}{f_{B}(t)} X_{t}, \quad t \in[T, \widetilde{T})$

Since the utility function $u$ is concave in $c$, the retirement benefits must be non-negative. Furthermore, we must have that $X_{\widetilde{T}}=0$, which ensures that all the savings that belong to the pension saver have been paid out during the distribution phase.

In both problems the functions $g_{A}(t)$ and $g_{B}(t)$ are of the same form. The first function has been defined by Richard (1975) as human capital and represents the expected present value of future earnings, or, in other words, what an individual's labor force is worth in the financial market. Function $g_{B}(t)$ represents the present value of the future premiums, i.e. the fraction of the human capital that 
is contributed to the pension savings account.

\section{Combined MSP and optimal control}

In this paper we further investigate a combined stochastic programming and stochastic optimal control approach that was originally presented in Geyer et al. (2009a). We cannot apply purely stochastic optimal control because the explicit solution can only be derived for simple models, which often cannot be applied in practice, and as soon as we add some realistic constraints, explicit solutions do not exist. In particular, we show in Sec. 5.1 that the optimal investment and sum insured derived by Richard (1975) is for a large variety of parameters problematic from a practical point of view. First, the optimal investment implies shorting the risk-free asset and gearing the mutual fund. Second, the sum insured is negative for most of the individual's lifetime. Third, while transaction costs have a minor impact on the optimal controls, the presence of taxes on capital gains affects the optimal investment strategy significantly.

To include the aforementioned constraints, one can choose the MSP approach, however, the main drawback of this approach is the limited ability to handle many time periods under sufficient uncertainty. The scenario tree grows exponentially with the number of time periods, and solving the problem soon becomes computationally intractable. For example, to deal with a long horizon one has

to choose long and increasing intervals between the decisions, see e.g. Carino et al. (1998), Dempster et al. (2003) and Consigli et al. (2012), add a steady state terminal value term to the objective function and approximate the infinite horizon problem using the dual equilibrium technique, see e.g. Grinold (1977, 1983) and Carino et al. (1998), or apply different scenario reduction algorithms, see e.g. Heitsch and Römisch (2009a b).

Each of the mentioned methods can handle the long time horizon problems, however, each method has also some drawbacks. For example, choosing long intervals between the stages implies that the individual does not have an opportunity to change her decisions for a considerable amount of time, and the optimal decisions may differ from those made more frequently. Furthermore, the impact of choosing increasing rebalancing intervals relative to constant intervals remains unknown. The dual equilibrium technique implies the approximation of the infinite horizon according to some assumed discount factor, and does not include the uncertainties during the steady state. Finally, despite the increasing research on scenario reduction algorithms, modelling the entire lifetime of an individual with yearly intervals still remains computationally intractable, unless one simplifies the tree during the later years to the branching factor of one. However, if the branching factor at any node is lower than the number of non-redundant assets, the scenarios may allow for arbitrage opportunities, which would affect the optimal decisions, see e.g. Kouwenberg (2001), Klaasen (2002) and Geyer et al. (2014).

In the light of these limitations, the mixed approach sounds appealing. It allows us to model the entire lifetime of the individual under realistic assumptions, with short intervals between the 


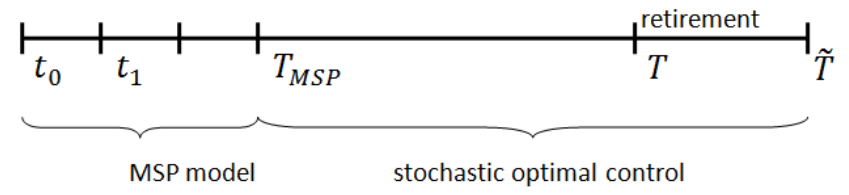

Figure 1: The first years decisions are solved by the MSP model, whereas the decision over the remaining lifetime of the individual are solved using HJB techniques.

decisions, and in a short computational time. The decisions are made under enough uncertainty both during the period modeled by MSP, and in the later years modeled by stochastic optimal control. Even though this approach assumes that the more realistic constraints are imposed only during the first years, and not the entire lifetime, the empirical results (Sec. 5) show that the impact of the constraints is visible even if they are imposed only for a short period of time, such as 6 years. We argue that the initial decisions are the most important because the customer needs an advice about her personal finance and pension at the present moment. A financial advisor or a pension fund typically would hold regular meetings with the customer and rerun the model for a different set of parameters than those initially chosen. This is necessary not only because the expectations about the economy change, but also because the customer might change her risk and impatience preferences. To what extend it is possible to neglect certain constraints and having a simpler model in the long run, is not trivial and left to future research.

To define the objective function for the mixed approach we split the lifetime of the individual into two periods: $t \in\left[t_{0}, T_{M S P}\right)$ and $t \in\left[T_{M S P}, \widetilde{T}\right)$. We apply the MSP approach during the first interval and stochastic optimal control during the remaining lifetime of the individual, see Fig. 1. The mathematical formulation for problem (A), defined in eq. (3. A ), can be rewritten using its recursive properties in the following way:

$V^{A}(t, x)=\sup _{\pi, c, I \in \mathcal{Q}\left[t, T_{M S P}\right)} E_{t, x}\left[\int_{t}^{T_{M S P}} e^{-\int_{t}^{s} \mu_{\tau} d \tau}\left(u\left(s, c_{s}\right)+\mu_{s} U\left(s, X_{s}+I_{s}\right)\right) d s+e^{-\int_{t}^{T_{M S P}} \mu_{\tau} d \tau} V^{A}\left(T_{M S P}, X_{T_{M S P}}\right)\right]$.

The above definition is convenient because it separates the problem into two intervals, which allows for applying different optimization methodologies in each interval. The crucial part of the model is to insert the optimal value function derived explicitly into the objective function of the MSP model, thus we can ensure that the MSP formulation incorporates the decisions for the entire lifetime and not only for the first years. We start with the latest period, $t \in\left[T_{M S P}, \widetilde{T}\right)$, and calculate the optimal value function at time $T_{M S P}$, i.e. $V^{A}\left(T_{M S P}, X_{T_{M S P}}\right)$ according to $4 \mathrm{~A}$. Then, we insert this function into eq. $8 \mathrm{~A}$ and solve the optimization problem using a multi-period stochastic model. In this way we construct one MSP formulation that covers the entire lifetime of the individual. We proceed 
analogously with problem (B). The mathematical formulation for the optimization problem defined in eq. $(3 \mathrm{~B})$ can be rewritten as

$$
V^{B}(t, x)=\sup _{\pi \in \mathcal{Q}\left[t, T_{M S P}\right), c \in \mathcal{Q}\left[\max (t, T), T_{M S P}\right)} E_{t, x}\left[\int_{\max (t, T)}^{T_{M S P}} e^{-\int_{t}^{s} \mu_{\tau} d \tau} u\left(s, c_{s}\right) d s+e^{-\int_{t}^{T_{M S P}} \mu_{\tau} d \tau} V^{B}\left(T_{M S P}, X_{T_{M S P}}\right)\right],
$$

where $V^{B}\left(T_{M S P}, X_{T_{M S P}}\right)$ is given in eq. $\left.4 \mathrm{~B}\right)$.

\section{MSP formulation}

The main elements of multi-stage stochastic programming are a scenario tree and an optimization model.

A scenario tree consists of nodes $n \in \mathcal{N}_{t}$ representing the range of possible outcomes for the uncertainties. All the nodes are uniquely assigned to stages $t=t_{0}, \ldots, T_{M S P}$ such that at the first stage there is a root node $n_{0}$, at the subsequent stages, $n \in \mathcal{N}_{t}, t>t_{0}$, each node has a unique ancestor $n^{-}$, and at all the stages except for the final stage, $n \in \mathcal{N}_{t}, t<T_{M S P}$, each node has children nodes $n^{+}$. The nodes at the final stage $T_{M S P}$ are called the leaves. A scenario $\mathcal{S}^{n}$ consists of a leaf $n$ and all its predecessors $n^{-}, n^{--}, \ldots, n_{0}$, or equivalently, a single branch from the root to the leaf. The number of scenarios in the tree equals the number of leaves. Each node has a probability $p r_{t, n}$, so that $\forall_{t} \sum_{n \in \mathcal{N}_{t}} p r_{t, n}=1$, and the probability of each scenario $\mathcal{S}^{n}$ is the product of the probabilities of all the nodes in the scenario, $p r_{\mathcal{S}^{n}}=p r_{T_{M S P}, n} \cdot p r_{T_{M S P}-1, n^{-}} \cdot p r_{T_{M S P}-2, n^{--}} \cdot \ldots \cdot p r_{t_{0}, n_{0}}$.

The optimal decisions along the tree are computed numerically at each node of the tree, given the information available at that point. Decisions do not depend on the future observations but anticipate possible future realizations of the random vector. After the outcomes have been observed, the decisions for the next period are made and depend both on the realizations of the random vector and the decisions made in the previous stage. This combination of anticipative and adaptive models in one mathematical framework makes this approach particularly appealing in financial applications; the investor can specify the composition of a portfolio by taking into account possible future movements of asset returns (anticipation), and rebalance the portfolio (take recourse decisions) as prices change, see Zenios (2008). The applications of MSP specifically in individual asset liability management can be found e.g. in Ziemba and Mulvey (1998), Kim et al. (2012) and Konicz and Mulvey (2013), whereas for a general introduction to stochastic programming we refer the reader to Birge and Louveaux (1997) and Ruszczyński and Shapiro (2003).

\subsection{Objective and constraints}

The first years decisions for the optimization problems considered in this paper are modeled by a $T_{M S P}$-period model. The decision variables are defined with respect to the nodes $n$ in the scenario 
tree, where $\mathcal{N}_{t}$ denotes the set of nodes corresponding to stage $t$, and $\mathcal{J}$ is the set of available financial assets. For each period $t=t_{0}, \ldots, T_{M S P}$, node $n \in \mathcal{N}_{t}$, and asset class $i \in \mathcal{J}$ we define the following decision variables and parameters:

\section{Parameters:}

$\begin{array}{ll}x_{0} & \text { initial value of the savings, } \\ l_{t} & \text { labor income/premiums paid to the savings account at time } t, \\ \mu_{t} & \text { mortality rate for a } y+t \text {-year old individual, } \\ q_{y+t} & \text { probability that a } y+t \text {-year old individual dies during the following period, } \\ q_{y+t}^{*} & \text { probability that a } y+t \text {-year old individual dies during the following period } \\ & \text { used for pricing life insurance/life annuities, } \\ p r_{t, n} & \text { probability of being at node } n, n \in \mathcal{N}_{t}, \text { obtained via scenario generation, see Sec. } 4.3 \\ r_{i, t, n} & \text { return on asset } i \text { at node } n \text { corresponding to stage } t, \text { obtained via scenario generation. }\end{array}$

\section{Decision variables:}

$\widetilde{P}_{i, t, n} \geq 0 \quad$ amount of asset $i$ purchased in period $t$ and node $n$, $\widetilde{S}_{i, t, n} \geq 0 \quad$ amount of asset $i$ sold in period $t$ and node $n$, $\widetilde{X}_{i, t, n} \quad$ holdings of asset $i$ at the beginning of period $t$, at node $n$, after rebalancing,

$\widetilde{X}_{t, n}^{-} \quad$ holdings in all assets at the beginning of period $t$, at node $n$, before rebalancing, $\widetilde{X}_{t_{0}, n_{0}}^{-}=x_{0}, \quad \widetilde{X}_{t, n}^{-}=\sum_{i \in \mathcal{J}}\left(1+r_{i, t, n}\right) \widetilde{X}_{i, t-1, n^{-}}, \quad t=t_{1}, \ldots, T_{M S P}, n \in \mathcal{N}_{t}$, $\widetilde{C}_{t, n} \quad$ consumption/benefit in period $t$ and node $n$, $\widetilde{I}_{t, n} \quad$ sum insured in period $t$ and node $n$ (problem (A) only).

We use capital letters with the tilde to denote the variables of the MSP formulation as opposed to the lowercase letters denoting the parameters for the model. Expression $\mathbb{1}_{\{(\cdot)=t\}}$ denotes an indicator function equal to 1 if $(\cdot)=t$ and 0 otherwise.

The entire MSP formulation that replicates the assumptions made in dynamic programming model setup, consists of the objective function and three constraints: the budget constraint, the asset inventory constraint and the constraint on positivity of certain variables. With only these three constraints we can replicate the continuous time models presented in Sec. 2. Afterwards, in Sec. 4.2, we modify these equations in order to investigate the impact of various factors, such as limits on portfolio composition, limits on the sum insured, transaction costs, and taxes on capital gains.

\subsubsection{Problem (A)}

The problem of optimizing the expected utility function of consumption and a terminal utility of leaving a positive amount of money upon death, where the investment, consumption and sum insured are the controlled processes, can be modeled with the set of the following equations. 
The objective function is obtained by substituting the integrals with the sums and the expectation operator $E$ with its discrete definition in the eq. $8 \mathrm{~A}$.

The budget constraint (10]A), or alternatively, the cash flow balance constraint specifies that the amount invested in the purchase of new securities plus consumption must be equal to the amount gained from the sale of the securities plus labor income that is paid to the savings account plus any initial savings $x_{0}$ that the person has at the beginning of the contract. Moreover, we add the term $q_{y+t}^{*} \widetilde{I}_{t, n}$ to the left hand side of the equation, which is the price for the life insurance that the investor pays at each period. The inclusion of the sum insured variable $\widetilde{I}_{t, n}$ in the budget constraint and in the objective function for problem (A) is an important part of the model that distinguishes our work from Geyer et al. (2009a).

Constraint 11A calculates the value of the savings. It is equal to the accumulated capital gains/losses on the assets held in portfolio from the previous period plus any amount purchased in the current period minus sold securities.

The continuous time versions of the problems assume the utility function $u$ to be strictly concave in $c$, and $U$ to be strictly concave in $X_{t}+I_{t}$. Furthermore, for $\gamma<0, u(0)=U(0)=-\infty$, therefore we define the positivity constraints $(12 \mathrm{~A})$. For $\gamma \in(0,1)$ we have $u(0)=0$ and $U(0)=0$, so the positivity constraints are substituted with non-negativity constraints.

This leads to the model:

$$
\begin{aligned}
& \max \sum_{s=t_{0}}^{T_{M S P}-1} \sum_{n \in \mathcal{N}_{s}} p r_{s, n} \cdot e^{-\int_{t_{0}}^{s} \mu_{\tau} d \tau}\left[u\left(s, \widetilde{C}_{s, n}\right)+q_{y+s} U\left(s, \widetilde{X}_{s, n}^{-}+\widetilde{I}_{s, n}\right)\right] \\
& +\sum_{n \in \mathcal{N}_{T_{M S P}}} p r_{T_{M S P}, n} \cdot e^{-\int_{t_{0}}^{T_{M S P}} \mu_{\tau} d \tau} \cdot V^{A}\left(T_{M S P}, \widetilde{X}_{T_{M S P}, n}^{-}\right), \\
& \sum_{i \in \mathcal{J}} \widetilde{P}_{i, t, n}+\widetilde{C}_{t, n}+q_{y+t}^{*} \widetilde{I}_{t, n}=x_{0} \mathbb{1}_{\left\{t=t_{0}\right\}}+\sum_{i \in \mathcal{J}} \widetilde{S}_{i, t, n}+l_{t}, \quad t=t_{0}, \ldots, T_{M S P}-1, n \in \mathcal{N}_{t}, \quad[10 \mathrm{~A}) \\
& \widetilde{X}_{i, t, n}=\left(1+r_{i, t, n}\right) \\
& \widetilde{C}_{t, n}>0, \quad \widetilde{X}_{i, t-1, n-1} \mathbb{1}_{\left\{t>t_{0}\right\}}+\widetilde{P}_{i, t, n}-\widetilde{S}_{i, t, n}, \quad t=t_{0}, \ldots, T_{M S P}-1, n \in \mathcal{N}_{t}, i \in \mathcal{J},(11 \mathrm{~A}) \\
& \widetilde{X}_{t, n}^{-}+\widetilde{I}_{t, n}>0,
\end{aligned}
$$

\subsubsection{Problem (B)}

The problem of maximizing the expected utility of retirement benefits, where the investment process is controlled both before and after retirement, whereas the benefits are controlled only after retirement, can be modeled using MSP formulation as follows. 
The objective function $(9 \mathrm{~B})$ is a discrete version of eq. $8 \mathrm{~B}$ where the integrals are substituted with the sums and the expectation operator $E$ with its discrete definition.

The budget constraint $110 \mathrm{~B}$ ) specifies that the amount invested in the purchase of new securities plus consumption must be equal to the amount gained from the sale of the securities plus premium that is paid to the savings account plus any savings $x_{0}$ that the person has at the beginning of the contract. We also add the term $q_{y+t}^{*} \widetilde{X}_{t, n}^{-}$to the right hand side of the balance equation, denoting the price that the pension fund pays the pension saver to be her only heir. Furthermore, we add the pension benefit to the side of the outgoing payments for $t \geq T$.

Constraint $11 \mathrm{~B}$ ) calculates the value of the savings on the pension account. This constraint is identical to the asset inventory balance for problem (A).

The utility function $u$ is assumed to be strictly concave in $c$, and for $\gamma<0$, we have $u(0)=U(0)=$ $-\infty$. Therefore, we assume that the benefits are positive, $(12 \mathrm{~B})$, or, for $\gamma \in(0,1)$, non-negative.

This leads to the following MSP formulation:

$$
\begin{aligned}
& \max \sum_{s=\max \left(t_{0}, T\right)}^{T_{M S P}-1} \sum_{n \in \mathcal{N}_{s}} p r_{s, n} \cdot e^{-\int_{t_{0}}^{s} \mu_{\tau} d \tau} u\left(s, \widetilde{C}_{s, n}\right) \\
& +\sum_{n \in \mathcal{N}_{T_{M S P}}} p r_{T_{M S P}, n} \cdot e^{-\int_{t_{0}}^{T_{M S P}} \mu_{\tau} d \tau} \cdot V^{B}\left(T_{M S P}, \widetilde{X}_{T_{M S P}, n}^{-}\right)
\end{aligned}
$$

$$
\sum_{i \in \mathcal{J}} \widetilde{P}_{i, t, n}+\widetilde{C}_{t, n} \mathbb{1}_{\{t \geq T\}}=x_{0} \mathbb{1}_{\left\{t=t_{0}\right\}}+\sum_{i \in \mathcal{J}} \widetilde{S}_{i, t, n}+l_{t}+q_{y+t}^{*} \widetilde{X}_{t, n}^{-}, \quad t=t_{0}, \ldots, T_{M S P}-1, n \in \mathcal{N}_{t}
$$

$\left.\widetilde{X}_{i, t, n}=\left(1+r_{i, t, n}\right) \widetilde{X}_{i, t-1, n^{-}} \mathbb{1}_{\left\{t>t_{0}\right\}}+\widetilde{P}_{i, t, n}-\widetilde{S}_{i, t, n}, \quad t=t_{0}, \ldots, T_{M S P}-1, n \in \mathcal{N}_{t}, i \in \mathcal{J}, 111 \mathrm{~B}\right)$

$\widetilde{C}_{t, n}>0$

$$
\left.t=\max \left(t_{0}, T\right), \ldots, T_{M S P}-1, n \in \mathcal{N}_{t} \cdot 12 \mathrm{~B}\right)
$$

\subsection{Additional constraints}

The reason for modelling the first years decisions with MSP is that this optimization method can easily capture the practical constraints such as limits on portfolio composition, limits on the sum insured, transaction costs, and taxes on capital gains. These constraints are commonly used in financial applications solved with MSP approach, see e.g. Geyer et al. (2009a) and Ferstl and Weissensteiner (2011), but rare in investment-consumption problems solved with stochastic optimal control. Below, we present how to modify the original constraints or add other constraints to the MSP formulation presented in Sec. 4.1. 
Limits on portfolio composition. A feature that is interesting from the point of view of a private investor, a pension saver and a pension fund, is the limit on the portfolio composition. For instance, no pension fund allows for borrowing or short selling of the assets. Alternatively, if the investor has certain preferences about the minimum and maximum percentage of her wealth invested in a certain asset, we would like to include it in the optimization model. The limits on the portfolio composition can be incorporated in the MSP formulation by adding the following constraints:

$\widetilde{X}_{i, t, n} \geq d_{i} \sum_{j \in \mathcal{J}} \widetilde{X}_{j, t, n}, \quad \widetilde{X}_{i, t, n} \leq u_{i} \sum_{j \in \mathcal{J}} \widetilde{X}_{j, t, n}, \quad t=t_{0}, \ldots, T_{M S P}-1, n \in \mathcal{N}_{t}, i \in \mathcal{J}$

where $d_{i}$ and $u_{i}$ are the lower and upper limits for the holdings of asset $i$. In particular, $d_{i}$ and $u_{i}$ can be extended to be time dependent, which would be suitable for a person with specific preferences only for a certain year.

Limits on the sum insured. Richard (1975)'s model does not assume any constraint on the size or the sign of the sum insured $I_{t}$. Including such a constraint in the dynamic programming approach is definitely not trivial to solve and to our knowledge the explicit solution for this case has not been derived. The MSP model allows for adding the limits on the sum insured in a straightforward way:

$\widetilde{I}_{t, n} \geq d_{i n s}, \quad \widetilde{I}_{t, n} \leq u_{i n s}, \quad t=t_{0}, \ldots, T_{M S P}-1, n \in \mathcal{N}_{t}$.

As shown later in Sec. 5.1, being able to control the sign of the sum insured is especially important from the practical point of view. A negative sum insured means that it is the individual who sells the life insurance to the pension fund for the price of $q_{y+t}^{*} \widetilde{I}_{t, n}$. Specifically, one should give up the part $\widetilde{I}_{t, n}$ of the savings upon death in return for the extra premium $q_{y+t}^{*} \widetilde{I}_{t, n}$. Theoretically, we could interpret this situation as investing a part of the savings in a life annuity, nevertheless, from a practical point of view having a negative sum insured sounds strange.

Transaction costs. Similarly, the presence of transaction costs $t c^{i}$ makes the considered problems more realistic. Investigating how the transaction costs affect the optimal controls is of practical importance. We add the costs as a percentage of the traded amount by modifying the budget equations $10 \mathrm{~A}$ and $(10 \mathrm{~B})$. We subtract the costs from the amount of the assets sold in a given period, and add the costs to the amount purchased. The modified budget equations are defined as follows:

$$
\begin{aligned}
& \sum_{i \in \mathcal{J}} \widetilde{P}_{i, t, n}\left(1+t c^{i}\right)+\widetilde{C}_{t, n}+q_{y+t}^{*} \widetilde{I}_{t, n}=x_{0} \mathbb{1}_{\left\{t=t_{0}\right\}}+\sum_{i \in \mathcal{J}} \widetilde{S}_{i, t, n}\left(1-t c^{i}\right)+l_{t}, \\
& \sum_{i \in \mathcal{J}} \widetilde{P}_{i, t, n}\left(1+t c^{i}\right)+\widetilde{C}_{t, n} \mathbb{1}_{\{t \geq T\}}=x_{0} \mathbb{1}_{\left\{t=t_{0}\right\}}+\sum_{i \in \mathcal{J}} \widetilde{S}_{i, t, n}\left(1-t c^{i}\right)+l_{t}+q_{y+t}^{*} \widetilde{X}_{t, n}^{-},
\end{aligned}
$$


for $t=t_{0}, \ldots, T_{M S P}-1$ and $n \in \mathcal{N}_{t}$.

Taxes on capital gains. Worth consideration are also taxes on capital gains $\tau^{i}$. Generating a scenario tree for the MSP model allows us to subtract taxes only from the positive capital gains, i.e.

${ }_{n e t} r_{i, t, n}= \begin{cases}r_{i, t, n}\left(1-\tau^{i}\right), & r_{i, t, n}>0 \\ r_{i, t, n}, & r_{i, t, n} \leq 0\end{cases}$

This requires changing the asset inventory balance constraints $(11 \mathrm{~A})$ and $(11 \mathrm{~B})$ as follows:

$\widetilde{X}_{i, t, n}=\left(1+{ }_{n e t} r_{i, t, n}\right) \widetilde{X}_{i, t-1, n^{-}} \mathbb{1}_{\left\{t>t_{0}\right\}}+\widetilde{P}_{i, t, n}-\widetilde{S}_{i, t, n}, \quad t=t_{0}, \ldots, T_{M S P}-1, n \in \mathcal{N}_{t}, i \in \mathcal{J}$.

$11 \mathrm{~A}, 11 \mathrm{~B})$

This is also convenient in contrast to the stochastic optimal control approach, where the asset prices

are modeled by the Black Scholes model, and one can only adjust the expected returns and volatility of the asset returns by introducing $\alpha_{i, n e t}=\alpha_{i}\left(1-\tau^{i}\right)$ and $\sigma_{i, n e t}=\sigma_{i}\left(1-\tau^{i}\right)$, see e.g. Bruhn (2013). The taxes are then subtracted from both positive (gains) and negative (losses) returns. The latter can be interpreted as a possibility to deduct the taxes from the negative capital income.

Other modifications. There are plenty of economic factors whose impact on the optimal control would be interesting to investigate but are beyond the scope of this paper. Probably the most relevant from a practical perspective is modelling the asset returns using a different model than Black Scholes. Geyer et al. (2009b) extended their previous work by applying the first-order unrestricted vector autoregressive process (VAR) to model asset returns. They find that there is a substantial difference in asset allocation which reflects the impact of time-varying investment opportunities, which, not surprisingly, shows the sensitivity of the model to the assumptions in the scenario generation.

\subsection{Scenario generation}

The uncertainty associated with the market returns is modeled by an $N$-1-dimensional random process. The multivariate return process evolves in discrete time, and the underlying probability distributions are approximated by discrete distributions in terms of a scenario tree.

We have tested a number of scenario generation methods for stochastic programming, including sampling, simulation, scenario reduction techniques and the methods based on matching the statistical properties of the underlying process, see e.g.Kaut and Wallace (2005) and Heitsch and Römisch (2009a). Since we combine the stochastic programming with the optimal control approach in one mathematical framework, our goal is to generate a scenario tree with the prices for the securities following the Black Scholes model defined in eq. (1). Specifically, we aim for constructing a multiperiod scenario tree with a discrete representation of a normal distribution $\mathcal{N}\left(\alpha_{i}-\sigma_{i}{ }^{2} / 2, \sigma_{i}\right)$, where 
the returns in two adjacent periods are independent and identically distributed. Therefore, the most suitable approach in our study is the moment matching method.

The moment matching approach has been first described in Høyland and Wallace (2001) and is based on solving a nonlinear optimization problem where both the asset returns and the probabilities of each node are the decision variables defining the scenario tree. This algorithm has later been improved in Høyland et al. (2003) where, instead of solving a nonlinear optimization problem, the authors suggest to perform a number of transformations that ensure that the required moments are matched. Since then, further improvements to this method have been made. Gülpınar et al. (2004) suggest a combined simulation and optimization approach. The asset returns are first simulated and then fixed in the optimization model, so that the only decision variables in the model are the probabilities associated with each node. This method can moreover be applied to generate the entire tree at once, and not node by node, as in the aforementioned approaches. Ji et al. (2005) is the first to propose a linear programming (LP) moment matching approach, though only for a single period tree. Similarly as in the previous paper, the outcomes of asset returns must be predetermined. Inspired by that work, Xu et al. (2012) design a new approach that combines simulation, the $K$ means clustering approach, and linear moment matching to generate the multi-stage scenario tree. This method ensures that the statistical properties are matched well, the generated scenario tree has a moderate size, the solution time is reduced, and at least two branches are derived from each nonleaf node. Finally, Chen and Xu (2013) improve this work by removing the simulation component and applying the $K$-means clustering method directly onto the historical dataset combined with LP moment matching. This approach significantly reduces the computational time while preserving the required statistical properties.

Despite the advantages of the newest scenario generation methods described above, for the purpose of our study we choose one of the older algorithms, namely Høyland and Wallace (2001), which we further improve by adding a priori the non-arbitrage constraints, see Klaasen (2002). This method matches the statistical properties of a geometric Brownian motion better than other algorithms in a situation when having as low a branching factor as possible is a priority. The algorithms described in $\mathrm{Xu}$ et al. (2012) and Chen and Xu (2013) are definitely more efficient and can capture more complex models for asset returns, such as the vector autoregressive and multivariate generalized autoregressive conditional heteroscedasticity models, but they require a larger branching factor. For our choice of assets and the distribution parameters, the satisfactory statistical match is obtained for a branching factor of at least 8, whereas the algorithm presented in Høyland and Wallace (2001) allows to generate the required scenario tree with only 4 branches. Our priority is to investigate the impact of some realistic constraints that are hard to implement in the stochastic optimal control approach, therefore to study more time periods, we choose a scenario tree with fewer branches. 


\section{$5 \quad$ Numerical results}

We first present realistic examples illustrating problems (A) and (B) in the original model setup (continuous time) and the solutions obtained by the MSP model presented in Sec. 4.1. Afterwards, we add or modify the particular constraints as described in Sec. 4.2 and investigate their impact on the optimal controls during the first years of the contract.

Parameters. If not specified in the captions of the tables and figures, the following parameters have been chosen for testing the models:

- Market: the number of assets, $N=3$; two risky and one risk-free asset, the expected rates of returns on the assets are, respectively, $\alpha_{1}=0.05, \alpha_{2}=0.07, r=\alpha_{3}=0.02$, the volatility of the assets, $\sigma_{1}=0.2, \sigma_{2}=0.25, \sigma_{3}=0$, and the correlation between the risky assets is $\operatorname{corr}_{12}=\operatorname{corr}_{21}=0.5$; all parameters are adjusted for inflation.

- Utility function: risk aversion, $1-\gamma=4$, corresponding to the optimal proportion in risky asset after retirement $\pi_{t}^{*}$ equal to $25 \%$, the impatience factor for the utility weights, $\rho=0.04$, intuitively chosen such that $\rho \geq r$, and the weight on the utility of bequest relatively to the utility of consumption, $\lambda=5$, implying that the optimal amount which the inheritors receive upon death is approximately equal to three and a half years of the person's consumption while she is alive, $X_{t}^{*}+I_{t}^{*} \approx 3.5 c_{t}^{*}(\lambda$ is only relevant for problem $(\mathrm{A})$ ).

- Contract: age at the beginning of the contract, $a g e_{0}=45$, retirement age, $a g e_{T}=65$, the final age at which the person is assumed to be dead with certainty, $\widetilde{T}=110$, type of retirement benefits (problem (B)): life annuity.

- Lifetime uncertainty: the mortality intensity model is of the form $\mu_{t}=\mu_{t}^{*}=\theta+10^{\beta+\delta(y+t)-10}$, where $y$ is the age of the person at time $t_{0}$, and $\theta, \beta$ and $\delta$ are constants ${ }^{3}$ The model has been calibrated to the mortality rates among Danish women in 2010 over age 40, Finanstilsynet (2010), where $\theta=0.0, \beta=4.59364, \delta=0.05032$. We approximate the probability that a $y+t$-year old individual dies during the next year by the mortality rate, i.e. $q_{y+t} \approx \mu_{t}$ and $q_{y+t}^{*} \approx \mu_{t}^{*}$.

- Scenario tree: number of MSP stages, $T_{M S P}=6$, branching factor in a single tree (number of nodes), $b f=4$, number of trees $n o^{\text {tree }}=50$, which implies in total $4^{5} \times 50=51,200$ scenarios.

- Cash-flows:

\footnotetext{
${ }^{3}$ In principle the mortality intensity model does not assume that an individual is dead at time $\widetilde{T}$ with probability 1. However, for $\widetilde{T}=110$, this error is negligible.
} 
- Problem (A). Labor income $l_{t}=27,000$ EUR, corresponding to the average Danish disposable income (after taxes) for a 45-year old individual, Danmarks Statistik (2010), average savings of a 45-year old individual, $x_{0}=60,000$ EUR,

- Problem (B). Before retirement: premiums, $l_{t}=4,000$ EUR, corresponding to $15 \%$ of the salary. Assuming that the person has been contributing to the pension account for around 15 years, accumulated with some capital gains, gives the value of the initial savings of $x_{0}=75,000 \mathrm{EUR}$; After retirement: premiums, $l_{t}=0$, the value of the savings is estimated from the expected savings for a 70 year old person (see before retirement case), i.e. $x_{0}=E\left[X_{25}^{B}\right]=225,000$ EUR.

Similarly as Geyer et al. (2009a), we have chosen to consider the small-scale optimization problems rather than the large-scale problems. On the first place, we are interested in replicating the optimal controls obtained from the continuous time models. The small-scale models are sufficient to evaluate whether a similar solution can be achieved by running the MSP model. With only three assets: one risk-free and two risky assets, the first four moments of a normal distribution can be approximated with 4 nodes. 6 periods with 4 nodes each give in total $4^{5}=1,024$ scenarios. Additionally, we rerun each program $n o^{\text {tree }}=50$ times for different scenario trees, and present the results in terms of means and standard errors from sampling. We use the notation $X_{t}^{*}, \pi_{t}^{*}, c_{t}^{*}$ and $I_{t}^{*}$ for the expected optimal value of savings, investment, consumption, and sum insured (average across all the scenarios) both from the Richard (1975)'s model and from the combined model.

Each problem has been implemented using Matlab 8.2.0.701 (R2013b) for calculation of the explicit solutions, GAMS 24.1.3 with CONOPT 3 (3.15L) solver for scenario generation, and GAMS with MOSEK 7.0.0.75 solver for the MSP formulation and solution of the stochastic programming models. The running time of each model for one scenario tree takes only a few seconds on a Dell computer with an Intel Core i5-2520M $2.50 \mathrm{GHz}$ processor and 4 GB RAM. Due to the linearity of the constraints, one can also approximate the objective function by a piecewise-linear interpolant, see

e.g. Kontogiorgis (2000) and Rasmussen (2011), and solve the problem using a linear solver. We have tested a number of both linear and nonlinear solvers, and we find that for the considered problems, in terms of speed, robustness and accuracy, MOSEK, which uses an interior point algorithm, is the best suited solver.

\subsection{Numerical results for problem (A)}

We start with analyzing the optimal controls obtained explicitly by solving Richard (1975)'s model and the MSP solutions replicating this model (no additional constraints). Fig. 2 (top left and right) shows the development in the expected values of the savings account, $X_{t}^{*}$, optimal consumption, $c_{t}^{*}$, and sum insured, $I_{t}^{*}$, in 1000 EUR (left figure), as well as the optimal proportion in both risky assets, $\pi_{t}^{*}$, and optimal proportion in the first risky asset $\pi_{t}^{* 1}$ (right figure) during the entire lifetime of the individual. The markers, which represent the optimal decisions obtained from the MSP 
model, closely follow the continuous lines representing the explicit solutions. Table 1 shows the exact numbers plotted on the figure for the first five years of the contract. Since we compare a continuous time model with a combined continuous and discrete time model, we expect the discrepancies. The explicit solutions are calculated based on the assumption that the labor income, consumption, sum insured, as well as capital gains occur simultaneously. In the MSP solutions we first account for the cash-flows, and then make the investment decisions based on the value of the savings after the cash-flows. Therefore, the investment control obtained in the MSP formulation is compared with the average continuous time investment over the two adjacent time periods. The standard deviations calculated from testing different scenario trees are negligibly small, which means that increasing the number of scenarios will not necessarily improve the precision of the results.

Richard (1975)'s model implies that the initial proportion in risky assets for our choice of parameters is given by $\pi_{t_{0}}^{*}=180 \%$, where the distribution between the risky assets is specified by the mutual fund theorem as $\pi_{t_{0}}^{* 1}=0.33 \pi_{t_{0}}^{*}$ and $\pi_{t_{0}}^{* 2}=0.67 \pi_{t_{0}}^{*}$. The optimal solution favours the asset with a higher return but also a higher volatility, therefore $\pi_{t}^{* 1}<\pi_{t}^{* 2}, \forall_{t}$. These proportions decrease smoothly over time as the individual manages to increase her savings due to labor income and capital gains. After retirement $\pi_{t}^{*}$ remains constant, which is a classical result known in the literature. The MSP solution closely follows the explicit solutions, though it consistently remains a few percentages below the original solutions. In particular, the MSP solution slightly favors the first risky asset (with a higher return and a higher volatility) and the ratio $\pi_{t}^{* 1} / \pi_{t}^{*}$ is 0.35 . The optimal consumption rate increases at a very slow pace over the entire lifetime. The increase is caused by the choice of impatience factor $\rho$ and risk tolerance $\gamma$ in relation to the choice of market parameters $\alpha_{i}, \sigma_{i}$, and the mortality rates, as explained later in the analysis of Figure 4 .

Interestingly, for our parameters choice the sum insured $I_{t}^{*}$ is positive only during the first year of the contract. Afterwards $I_{t}^{*}$ is negative, which is also consistent with Richard (1975) conclusions. Recall that the amount paid out to the inheritors upon the person's death is equal to the value of the savings plus the sum insured. Therefore $I_{t}^{*}$ can be negative as long as $X_{t}^{*}+I_{t}^{*}>0$. The size and the sign of sum insured depends on parameter $\lambda$. Choosing $\lambda=1$ means that the individual puts an equal weight on her consumption and the bequest motive, therefore the optimal legacy amount is approximately equal to the optimal consumption while the person is alive, i.e. $X_{t}^{*}+I_{t}^{*} \approx c_{t}^{*}$. Fig. 2 and Table 1 show the case for $\lambda=5$, where the optimal death sum corresponds to approximately three and a half years of optimal consumption while the person is alive, i.e. $X_{t}^{*}+I_{t}^{*} \approx 3.5 c_{t}^{*}$.

The example takes as input some realistic parameters, however, some of the optimal controls are problematic from a practical point of view. First, the proportion in risky assets is higher than $100 \%$ for $t \leq t_{3}$. It may happen that the individual does not have an opportunity to borrow money and short the risk-free asset. Second, the sum insured is negative for $t>t_{0}$. Thus, rather than to purchase a life insurance from the pension company, the individual should sell a life insurance to the pension company. Specifically, she should give up the part $I_{t}^{*}$ of her savings upon death in return for the extra premium $q_{y+t}^{*} I_{t}^{*}$. Theoretically, we could interpret this situation as investing a part of the 

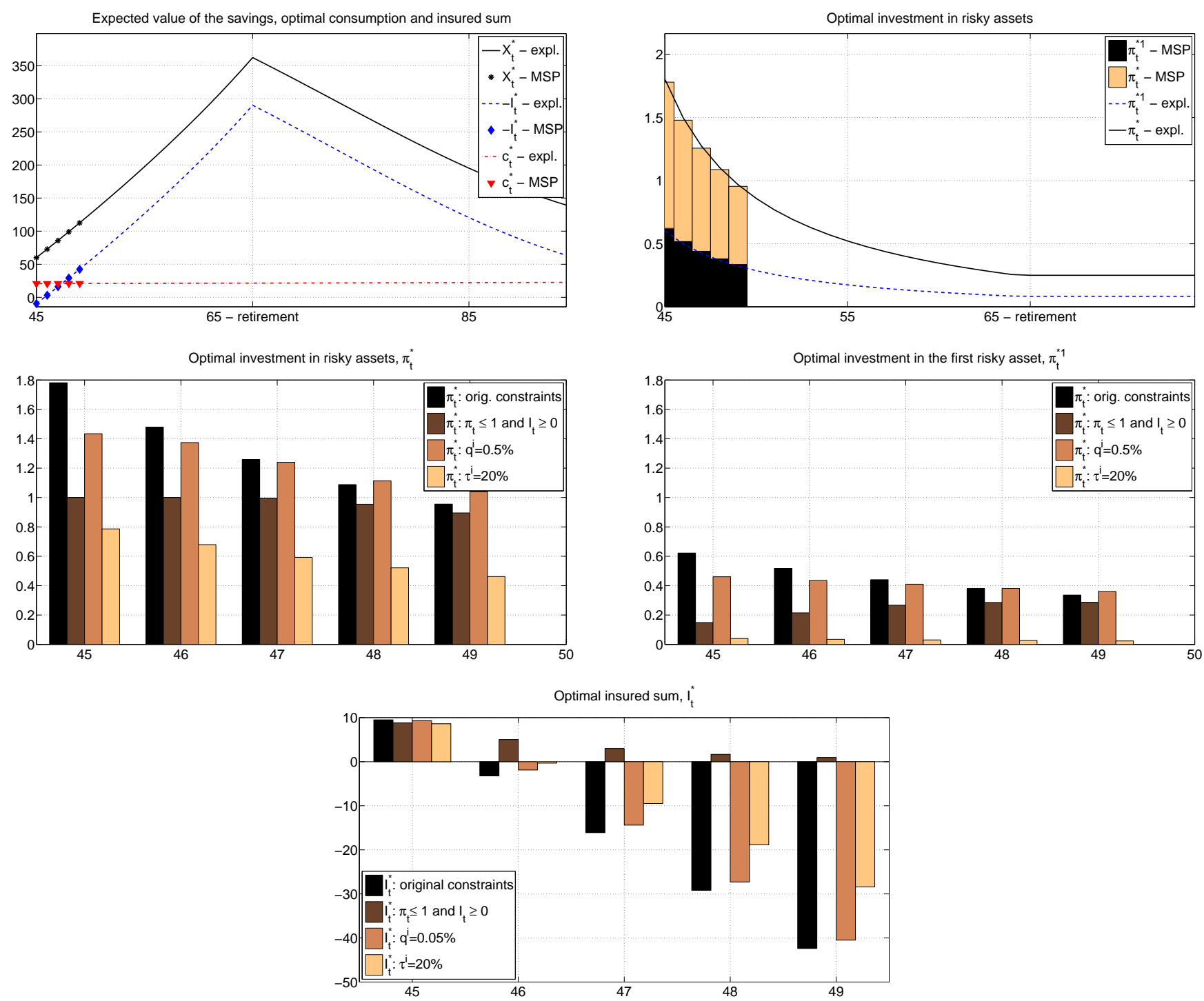

Figure 2: Problem (A). Explicit solution and MSP solution for the expected value of the savings, optimal consumption and sum insured (left, top, in 1000 EUR). Optimal investment in both risky assets and in the first risky asset (right, top, $\left.\pi_{t}^{*}=\pi_{t}^{* 1}+\pi_{t}^{* 2}\right)$. The MSP solution for the expected optimal investment in both risky assets (left, middle) and in the first risky asset (right, middle) given different constraints. The MSP solution for the expected optimal sum insured given different constraints (bottom, in 1000 EUR).

savings in a life annuity, nevertheless, from a practical point of view, having a negative sum insured sounds strange.

We have shown that the closed-form solutions can be replicated well and the patterns and the level of the optimal controls are consistent with the continuous time models. Therefore, we find the model reliable and use it as a tool to analyze the optimal controls under the effects of various modifications during the first years of the contract. 
A borrowing constraint and a positive sum insured constraint. Figure 2 (middle left and right, and bottom plot) and Table 1 show the optimal solutions that simultaneously account for two additional constraints: (i) the individual does not have a possibility to borrow money in order to invest more than she has in other assets (this constraint is defined in eq. $(16)$ where $u^{i}=100 \%$ ), and (ii) the individual is not allowed to sell the life insurance to the pension fund, $I_{t}>0$, as defined in eq. (17). As expected, the constraints directly affect the optimal investment and sum insured. The optimal investment $\pi_{t}^{*}$ is now equal to its upper limit $100 \%$ and decrease faster than in the original model setup. The constraint naturally also implies a change in the distribution between the risky assets. Before, the ratio of the first risky asset to the total risky investment was given by $\pi_{t}^{* 1} / \pi_{t}^{*} \approx 0.35$. With the new constraints the ratio starts with 0.15 at $t_{0}$ and with time converges to the original optimal ratio, which implies that $\pi_{t}^{* 1}$ increases until the optimal ratio between the risky assets is reached. The optimal sum insured is positive and converges to its lower limit 0. Finally, the value of the savings, and as follows, the optimal consumption, is lower, due to more conservative investment and positive costs for the sum insured $q_{y+t}^{*} I_{t}$.

Transaction costs. Secondly, we investigate the effect of transaction costs $t c^{i}$. These have been defined as a constant percentage of a traded amount and are the same for purchases and sales and for all assets, see eq. (10[A]). As a result, the amount invested in risky assets is lower during the first few years than in the original problem, but after a few years stays above the original $\pi_{t}^{*}$. We conclude that introducing the transaction costs implies that the development of the risky investment over time is smoother, i.e. it decreases as in all other cases but the difference between the maximum and minimum value is smaller. We explain it by the fact that rebalancing portfolio is expensive. A smoother optimal investment implies the lower traded amount, hence lower costs. Other optimal controls, i.e. consumption and sum insured, are not much affected by the transaction costs. As well as the total savings, the optimal values are slightly lower (lower absolute value for the sum insured). Fig. 2 (middle, left and right) shows the results for $t c^{i}=0.5 \%$, but we have also investigated higher costs, $t c^{i}=1 \%$, and the patterns for the optimal controls remain similar.

Taxes on capital gains. Finally, we investigate the impact of taxes on capital gains on the optimal decisions. We have integrated a linear taxation on the positive returns on asset $i, \tau^{i}$, as defined in eq. (18). In comparison with other constraints, taxes on capital gains are the most influential for the optimal decisions. Only $\tau^{i}=20 \%$ causes already a loss of $15 \%$ of the expected savings after 5 years relatively to the original problem. Less savings implies less money to consume and to purchase a life insurance. Both the consumption and the absolute value of the sum insured decrease, but the biggest impact can be seen on the investment controls. The optimal percentage in stocks is only $\pi_{t_{0}}^{*}=80 \%$ comparing to the original $\pi_{t_{0}}^{*}=178 \%$, while the proportion in the first risky asset is only $\pi_{t_{0}}^{* 1}=4 \%$. Subtracting taxes only from the positive returns makes the scenario tree asymmetric and favours the risk-free asset (the positive outcomes are lower, whereas the negative outcomes are as severe as they 
were before). A similar conclusion has been drawn by Geyer et al. (2009a). The MSP solution is also more volatile for this case than for other considered constraints; the investment controls vary within $3-8 \%$ for different scenario trees.

\subsection{Numerical results for problem (B)}

Problem (B) is relevant for a pension product design in a defined contribution pension plan. While in certain products (such as unit-linked products) the individual can decide on the investment strategy both before and after retirement, except for choosing the type of the benefits (lump sum payment, temporary or life long benefits), she cannot control control how the benefits will be distributed. This decision belongs to the pension fund. However, the pension fund can take into consideration the pension saver's risk preferences $\gamma$, the market parameters hidden under the variable $\varphi$, the impatience factor $\rho$ and both regular and pricing mortality intensities $\mu_{t}$ and $\mu_{t}^{*}$, and design the payout profile such that the individual's preferences are included.

Optimal annuity concept. The optimal pension benefits are of the form $c_{t}^{*}=\frac{w_{t}}{f_{B}(t)} X_{t}$, see eq. (7B). Inserting the definitions of functions $w_{t}$ and $f_{B}(t)$ leads to

$c_{t}^{*}=\frac{X_{t}}{\bar{a}_{y+t}}, \quad t \in[T, \widetilde{T})$

where $\bar{a}_{y+t}$ is a traditional single-life annuity that pays 1 EUR per year continuously to an individual who is $y+t$ years old, i.e.

$\bar{a}_{y+t}=\int_{t}^{\widetilde{T}} e^{-\int_{t}^{s}\left(\bar{r}+\bar{\mu}_{\tau}\right) d \tau} d s$,

where

$\bar{r}=\frac{1}{1-\gamma} \rho-\frac{\gamma}{1-\gamma} \varphi, \quad \varphi=r+\frac{(\alpha-r)^{2}}{2 \sigma^{2}(1-\gamma)}, \quad \bar{\mu}_{\tau}=\frac{1}{1-\gamma} \mu_{\tau}-\frac{\gamma}{1-\gamma} \mu_{\tau}^{*}$.

Parameters $\bar{r}$ and $\overline{\mu_{t}}$ are the utility adjusted interest rate and mortality rate, respectively.

Figures 3 and 4 show the expected optimal benefits $c_{t}^{*}$ for the individuals with different risk aversion $1-\gamma$ and impatience factor $\rho$. Fig. 3 shows the initial value of the benefit at time of retirement, $c_{T}^{*}$, and its sensitivity on $\gamma$ and $\rho$. Given the value of the savings account $X_{T}=265,000$ EUR and no premiums after retirement, the size of the benefits for $t=T$ varies between 12, 800 EUR and 19,500 EUR. The lowest initial benefit is for patient persons who prefer more risky investment, i.e. those characterized by f.ex. $\rho=0.0$ and $\gamma=0$, whereas the highest benefits will be received still by those who prefer more risky investment but are impatient, i.e. $\rho=0.04$ and $\gamma=0$. The tendency is similar for the risk averse retirees - the impatient ones will receive the highest annuities in the beginning of the retirement. 


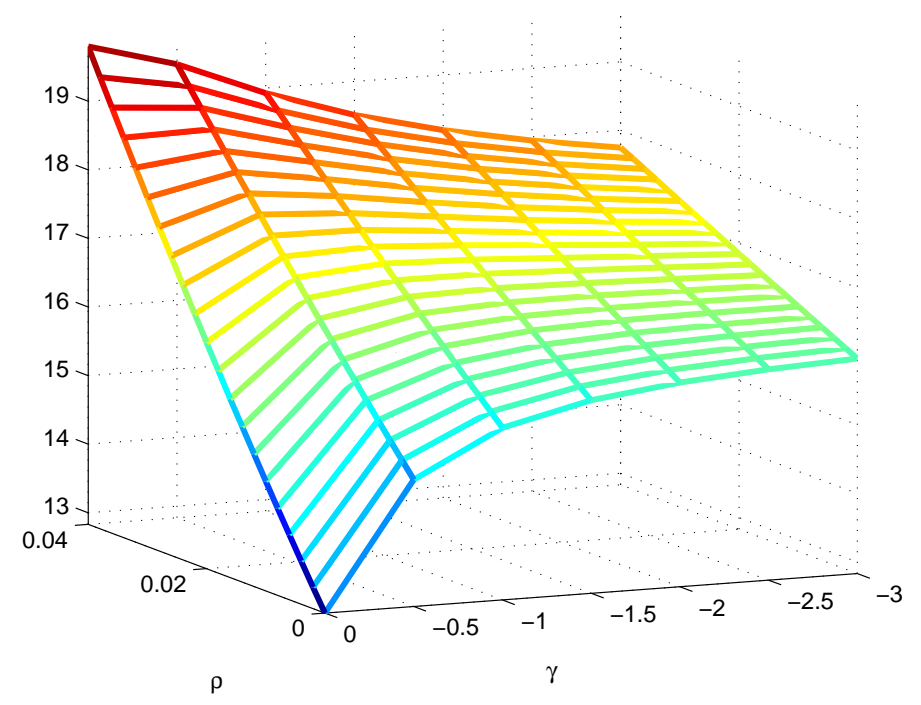

Figure 3: Sensitivity of the optimal benefits at the beginning of retirement $c_{T}^{*}$ to risk aversion $1-\gamma$ and impatience factor $\rho$. The numbers are in 1000 EUR. $x_{T}=265$. Other parameters are as specified in Sec. 5 .
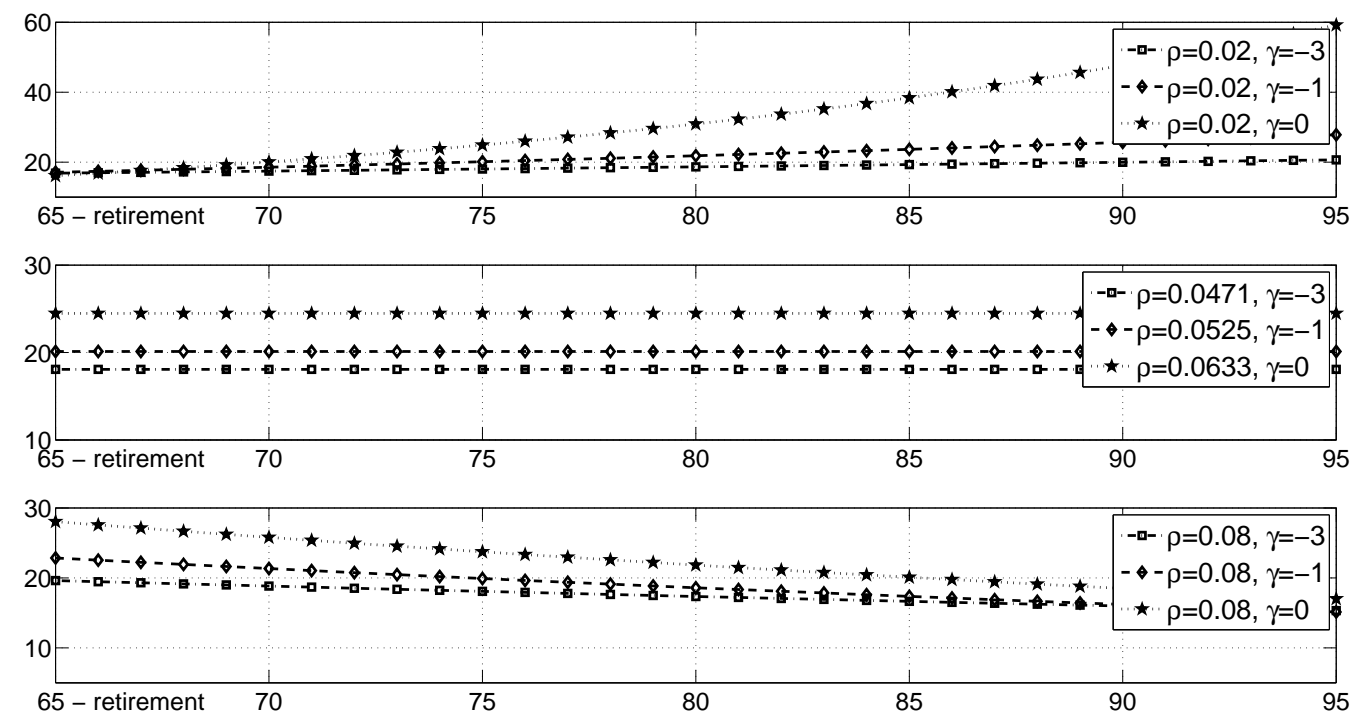

Figure 4: Optimal benefits $c_{t}^{*}$ for the first 30 years of retirement. Sensitivity of the benefits to various choices of the impatience factors and risk preferences. The numbers are in 1000 EUR. $x_{T}=265$. Other parameters are as specified in Sec. 5

Fig. 4 shows the development of the size of the benefits during retirement (the payout profile) for different values of $\gamma$ and $\rho$. We immediately notice that the optimal payout profile is different for individuals with different patience levels and risk preferences. The annuity payments can be constant, decreasing or increasing. To be precise, it is the value of the impatience factor $\rho$ relatively 
to the average return on investment adjusted for the risk aversion level $1-\gamma$ that drives the wealth process, which controls the development of the payments. Impatient persons (f.ex. with $\rho=0.08$ ) wish to receive the highest benefits in the first years of the retirement, whereas those more patient $(\rho=0.02)$ receive lower benefits in the beginning. The latter allows for larger capital gains during retirement, thus on average the benefits during the individual's older years are higher than upon retirement. Parameter $\gamma$ affects mostly the size of the benefits. The more risky investment gives on average a higher return, thus higher benefits. Furthermore, risk averse persons (f.ex. $\gamma=-3$ ) will receive each year smoother benefits (the difference between $c_{65}^{*}$ and $c_{95}^{*}$ is smaller) than a person with a low risk aversion, such as $\gamma=0$.

Basic constraints. We present the results for problem (B) for the time before retirement $t<T$ and after retirement $t \geq T$ on Fig. 5 and Table 2, Fig. 5 shows the development in the expected values of the savings account $X_{t}^{*}$ and optimal benefits $c_{t}^{*}$ (in 1000 EUR) before retirement (top, left) and after retirement (middle, left). The figures on the right show the optimal proportion in the first risky asset $\pi_{t}^{* 1}$ during the lifetime of the individual for the case before retirement (top, right) and after retirement (middle, right). Given that at age 45 the individual has $x_{0}=75,000$ EUR on her savings account and she continues to pay the premiums of $l_{t}=4,000$ EUR every year, which are invested in a conservative way, i.e. $\gamma=-3$, she should expect that her savings at retirement will reach 265,000 EUR, which will define the pension benefits at almost $c_{t}^{*}=18,000 \mathrm{EUR}$ at age 65 . The size and development of the benefits is optimal given she puts more weight on the benefits just after retirement than on those in the future, and given she is risk averse, i.e. $\rho=0.04$ and $\gamma=-3$. The optimal investment is more conservative than in problem (A) because it is proportional to the sum of the value of the savings and the present value of the pension contributions relatively to the value of savings, see eq. (7B). Since the pension contributions $l_{t}$ are much lower than the labor income in problem (A), $\pi_{t}^{*}$ is much lower than in problem (A) starting with $45 \%$ at age 45 and decreasing afterwards. After retirement, the optimal proportion in risky assets remains constant at $25 \%$. The ratio of the investment in the first risky asset to the total risky assets according to the mutual fund theorem remains the same, i.e. $\pi_{t}^{* 1} / \pi_{t}^{*}=33 \%$, both before and after retirement.

Similarly as in problem (A) we conclude that the MSP solution closely follows the optimal controls derived explicitly. The model provides a realistic solution that can be applied by a pension fund. Nevertheless, there is a number of constraints whose effect on the optimal control is worth investigating. Incorporating the pension saver's preferences such as the limits on the portfolio composition, or other practical constraints such as transaction costs and taxes on capital gains using stochastic optimal control is not trivial, therefore, to investigate the desired factors and to add flexibility to the model, we choose the mixed approach.

Limits on portfolio composition. Imagine a 70 year old pension saver who simply is not satisfied with the way the pension fund invests her savings. In particular, she believes that the first risky 

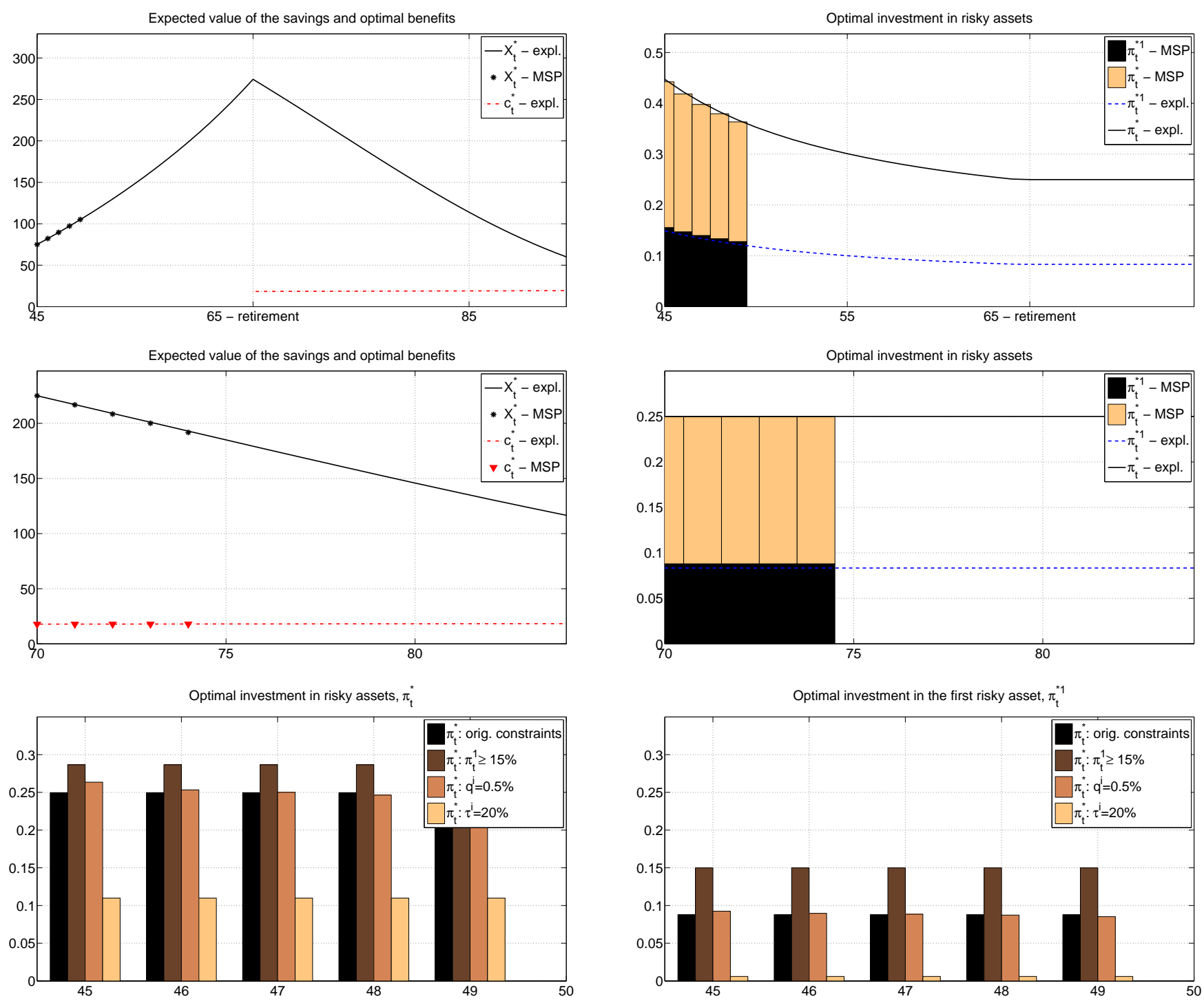

Figure 5: Problem (B) before and after retirement. Explicit solution and MSP solution for the expected value of the savings and optimal benefits before retirement (left, top, in $1000 \mathrm{EUR}$ ). Explicit solution and MSP solution for the expected value of the savings and optimal benefits after retirement (left, middle, in 1000 EUR). Optimal investment in risky assets before retirement (right, top) and after retirement (right, middle). The MSP solution for the expected optimal investment after retirement given different constraints in both risky assets (bottom, left) and in the first risky asset (bottom, right). $\pi_{t}^{*}=\pi_{t}^{* 1}+\pi_{t}^{* 2}$. 
asset is more valuable and does not agree that after retirement only $9 \%$ of her savings are invested in this asset (see Table 2, original constraints). She prefers a higher percentage in the first risky asset and defines her new preferences, for instance, as minimum 15\% should be invested in the first risky asset. Such a constraint can easily be incorporated in the MSP model by adding eq. (16), where constant $d_{1}$ is set to be equal $15 \%$. The optimal solution for this case is shown on Figure 5 (middle left and right) and Table 2, Both the total investment in risky assets and the investment in each of the risky assets remain constant as before. Though, both values are now higher. The pension saver's preferences are taken into account and $\pi_{t}^{* 1}$ raises from $9 \%$ in the original model setup to $15 \%$, whereas the investment in both risky assets increases from $25 \%$ to $29 \%$. The fact the $\pi_{t}^{*}$ increases as well can be explained by the fact that there exists an optimal ratio between the risky assets which the model tries to remain as closely as possible.

The MSP formulation allows for adding various limits on the portfolio. The limits can be both from above and below, and separately for each asset class. We have investigated various combinations of limits on a particular asset, and without presenting the numbers, we came to the following conclusions. None of these constraints have much effect on the overall value of the savings or the optimal benefits, but adding a limit just to one asset class directly affects the distribution between all three asset classes. Imposing a higher percentage on an asset class than the percentage given by the optimal control in the case without constraints, results in setting this asset exactly to this limit. And, vice versa, limiting the amount of savings in a given asset class from above when the original optimal investment suggests a higher amount, results in new optimal investment equal to the limit. Furthermore, the limit set on one of the risky assets, triggers the change in the same direction of the percentage of the second risky assets.

Transaction costs. In problem (A) we concluded that adding transaction costs, defined as a percentage of a traded amount, implies a smoother development in the proportion invested in risky assets. In this case, where the original optimal investment is constant, the development of $\pi_{t}^{*}$ is no longer constant, but actually slowly decreases. The reason for that is that while the value of the savings decreases, trying to rebalance the portfolio each year to a constant proportion is more expensive than rebalancing to the decreasing proportion. As before, the changes in the optimal benefits are so small that it is hard to conclude how the transaction costs affect the benefits, except for the obvious fact, that the savings are slightly lower, which implies lower benefits.

Taxes on capital gains. Finally, to strengthen the conclusions we drew from investigating the effect of adding taxes on the positive returns in problem (A), we repeat this investigation for problem (B). The results are similar: the impact of taxes on the optimal investment is significant. Without taxes the optimal investment in both risky assets was given $\pi_{t}^{*}=25 \%$ and in the first risky asset $\pi_{t}^{* 1}=9 \%$. With the presence of taxes, the optimal controls are down to $\pi_{t}^{*}=11 \%$ and $\pi_{t}^{* 1}=1 \%$. Again, we explain this fact by the asymmetry in the scenario tree caused by subtracting the taxes only 
from the positive capital gains. It is interesting though that taxes affect the size of the investment but not its development, i.e. the proportions remain constant as in the case of the original problem. Furthermore, the MSP solution is not as volatile as for problem (A). The standard deviations are low, which shows a stability for different scenario trees. In terms of other controls, similarly as in problem (A), the presence of taxes significantly affects the value of the savings, implying lower and decreasing pension benefits.

\section{Conclusions and future work}

We have presented a model combining two optimization technologies: multi-period stochastic programming and dynamic programming, to obtain optimal decisions related to various problems within personal finance and retirement. The optimization methods complement each other well. MSP allows for building flexible and practical models, which are difficult to solve with the stochastic optimal control approach. The explicit formulas, however, can handle any number of periods and are useful for the validation of the results obtained by the MSP. The considered problems are solved using a nonlinear solver.

The numerical results show that among the considered limits on portfolio composition, limits on the sum insured, transaction costs and taxes on capital gains, the presence of taxes affects the optimal controls the most, and the changes in both the value of savings and the controlled processes are significant.

Except for the contribution regarding computational methods, the paper introduces an important aspect regarding designing pension benefits in a defined contribution pension scheme. Problem (B) suggests how the pension fund could decide on the initial size of the benefits and their development during the decumulation phase so that the individual's risk and impatience preferences are captured. Interestingly, the pension saver's preferences have a higher impact on the size and development of the benefits than limits on portfolio composition, transaction costs or taxes on capital gains.

The model can be improved in various ways. The most interesting would be the extension of the MSP part to even more periods while still keeping the running time of the program within a couple of minutes. Another suggestion is to introduce the bond market instead of the risk-free asset and/or the uncertainty on the labor income/premiums. Finally, since the stochastic programming approach allows the objective function to take a variety of forms, it could be of interest to consider a different utility function such as loss aversion framework, where an individual can control both the gains and losses in savings relative to a pre-defined target, or a multi-objective function, which takes into account other preferences than risk aversion and impatience. 


\section{A Application of the mutual fund theorem}

The mutual fund theorem has been originally defined in Merton (1971) for an individual with a known lifetime who wishes to maximize the expected utility of consumption. Richard (1975) extended this result and proved that the theorem obtains also for an investor with the uncertain time of death optimizing the utility of consumption and bequest.

Theorem 1 (Merton (1971), p. 384). Given n assets with prices $S_{i}$ whose changes are log-normally distributed, then (1) there exists a unique pair of "mutual funds" constructed from linear combinations of these assets such that, independent of preferences (i.e., the form of utility function), wealth distribution, [probability distribution of lifetime or life insurance opportunities, Richard (1975), $p$. 194], or time horizon, individuals will be indifferent between choosing from a linear combination of these two funds or a linear combination of the original $n$ assets.

Corollary 1 (Merton (1971),p. 386). If one of the assets is "risk-free" (say the Nth), then one mutual fund can be chosen to contain only the risk-free security and the other to contain only the risky assets in the proportions:

$\forall_{i=1, \ldots, N-1} \quad \theta_{i}=\frac{\sum_{j=1}^{N-1}\left[\sigma_{i j}\right]^{-1}\left(\alpha_{j}-r\right)}{\sum_{i=1}^{N-1} \sum_{j=1}^{N-1}\left[\sigma_{i j}\right]^{-1}\left(\alpha_{j}-r\right)}$,

where $\left\{\alpha_{i}, \sigma_{i j}\right\}$ define the physical distribution of the returns, $\sigma_{i j}=\sigma_{i} \sigma_{j} \operatorname{corr}_{i j}$, and corr $_{i j}$ is the correlation coefficient between assets $i$ and $j$.

Hence, without loss of generality, we can work with only two assets, one risk-free and the other a mutual fund of risky assets with a log-normally distributed price $S_{t}$ defined by

$d S_{t}=\alpha S_{t} d t+\sigma S_{t} d W_{t}$

where

$\alpha=\sum_{i=1}^{N-1} \theta_{i} \alpha_{i}, \quad \sigma^{2}=\sum_{i=1}^{N-1} \sum_{j=1}^{N-1} \theta_{i} \theta_{j} \sigma_{i j}, \quad d W=\sum_{i=1}^{N-1} \theta_{i} \frac{\sigma_{i}}{\sigma} d W_{i}$.

We assume in our study one risk-free asset and a mutual fund consisting of $N-1$ risky assets. The optimal proportion between the risk-free asset and the mutual fund during the period $\left[T_{M S P}, \widetilde{T}\right)$ is calculated explicitly using stochastic optimal control (Hamilton-Jacobi-Bellman techniques). Thereafter, we apply the mutual fund theorem to find the optimal allocation between the risky assets in the mutual fund.

\section{B Derivation of the optimal controls for problem (B)}

We derive the optimal value function and the optimal controls for the problem of maximizing the utility of consumption, where the control processes are specified over different time periods: investment is controlled both before and after retirement, $\pi_{t} \in \mathcal{Q}\left[t_{0}, \widetilde{T}\right)$, and consumption is controlled only after retirement, $c_{t} \in \mathcal{Q}\left[\max \left(t_{0}, T\right), \widetilde{T}\right)$. The problem is solved first for the period after retirement, where we refer to Bruhn and Steffensen (2011), and then for the period before retirement, which is 
one of the contributions of this paper. In the latter we insert the optimal value function obtained in after retirement period as the boundary condition at time $T$.

After retirement period. The problem of optimizing expected utility of consumption after retirement in the case where the person controls investment $\pi_{t}^{*}$, life insurance $I_{t}^{*}$, and consumption $c_{t}^{*}$, and has no bequest motive, has been solved in Bruhn and Steffensen (2011). The authors derive the optimal value function

$V(t, x)=\frac{1}{\gamma} f_{t}^{1-\gamma} x^{\gamma}$,

and the explicit formulas for the optimal controls

$\pi_{t}^{*} X_{t}=\frac{\alpha-r}{\sigma^{2}(1-\gamma)} X_{t}, \quad c_{t}^{*}=\frac{w_{t}}{f_{t}} X_{t}, \quad I_{t}^{*}=0$,

where

$f_{t}=\int_{t}^{\widetilde{T}} e^{-\frac{1}{1-\gamma} \int_{t}^{s}\left(\mu_{\tau}-\gamma\left(\mu_{\tau}^{*}+\varphi\right)\right) d \tau} w_{s} d s, \quad \varphi=r+\frac{(\alpha-r)^{2}}{2 \sigma^{2}(1-\gamma)}$.

Before retirement period. We apply Hamilton-Jacobi-Bellman techniques to solve the problem. We first define the HJB equation for the considered period, based on the savings dynamics defined in eq. $(2 \mathrm{~B})$ :

$\frac{\partial V(t, x)}{\partial t}-\mu_{t} V+\sup _{\pi}\left\{l_{t} \frac{\partial V(t, x)}{\partial x}+\left(r+\pi_{t}(\alpha-r)+\mu_{t}^{*}\right) x \frac{\partial V(t, x)}{\partial x}+\frac{1}{2} \pi_{t}^{2} \sigma^{2} x^{2} \frac{\partial^{2} V(t, x)}{\partial x^{2}}\right\}=0$,

$V(T, x)=\frac{1}{\gamma} f_{T}^{1-\gamma} X_{T}^{\gamma}$.

The boundary condition is equal to the optimal value function at time $T$ given by eq. (20).

To solve the problem we first guess the solution and verify that it is a correct guess:

$V(t, x)=\frac{1}{\gamma} f_{t}^{1-\gamma}\left(x+g_{t}\right)^{\gamma}$.

We find the functions $f_{t}$ and $g_{t}$ by plugging in the derivatives to the HJB equation:

$$
\begin{aligned}
& \frac{1-\gamma}{\gamma} f_{t}^{-\gamma} \frac{\partial f_{t}}{\partial t}\left(x+g_{t}\right)^{\gamma}+f_{t}^{1-\gamma}\left(x+g_{t}\right)^{\gamma-1} \frac{\partial g_{t}}{\partial t} \\
& =\mu_{t} \frac{1}{\gamma} f_{t}^{1-\gamma}\left(x+g_{t}\right)^{\gamma}-l_{t} f_{t}^{1-\gamma}\left(x+g_{t}\right)^{\gamma-1} \\
& -\left(r+\mu_{t}^{*}\right)\left(x+g_{t}\right) f_{t}^{1-\gamma}\left(x+g_{t}\right)^{\gamma-1}+\left(r+\mu_{t}^{*}\right) g_{t} f_{t}^{1-\gamma}\left(x+g_{t}\right)^{\gamma-1}-\frac{1}{1-\gamma} \frac{(\alpha-r)^{2}}{2 \sigma^{2}} f_{t}^{1-\gamma}\left(x+g_{t}\right)^{\gamma},
\end{aligned}
$$

and obtain for $t \in\left[t_{0}, T\right)$ :

$$
\begin{aligned}
& g_{t}=\int_{t}^{T} e^{-\int_{t}^{s}\left(r+\mu_{\tau}^{*}\right) d \tau} l_{s} d s \\
& f_{t}=e^{-\frac{1}{1-\gamma} \int_{t}^{T}\left(\mu_{\tau}-\gamma\left(\mu_{\tau}^{*}+\varphi\right)\right) d \tau} f_{T}=e^{-\frac{1}{1-\gamma} \int_{t}^{T}\left(\mu_{\tau}-\gamma\left(\mu_{\tau}^{*}+\varphi\right)\right) d \tau} \int_{T}^{\widetilde{T}} e^{-\frac{1}{1-\gamma} \int_{T}^{s}\left(\mu_{\tau}-\gamma\left(\mu_{\tau}^{*}+\varphi\right)\right) d \tau} w_{s} d s .
\end{aligned}
$$


The optimal investment is then given by:

$\frac{\partial}{\partial \pi}: \quad \pi_{t}^{*}=-\frac{\alpha-r}{\sigma^{2} x} \frac{\frac{\partial V(t, x)}{\partial x}}{\frac{\partial^{2} V(t, x)}{\partial x^{2}}}=\frac{1}{1-\gamma} \frac{\alpha-r}{\sigma^{2}} \frac{x+g_{t}}{x}$.

\section{References}

Barro, D. and Canestrelli, E. (2005). Dynamic portfolio optimization: Time decomposition using the maximum principle with a scenario approach. European Journal of Operational Research, 163(1):217-229.

Barro, D. and Canestrelli, E. (2006). Time and nodal decomposition with implicit non-anticipativity constraints in dynamic portfolio optimization. Mathematical Methods in Economics and Finance, $1: 1-20$.

Barro, D. and Canestrelli, E. (2011). Combining stochastic programming and optimal control to solve multistage stochastic optimization problems. Technical Report 2011_24, Department of Economics, University of Venice Ca' Foscari".

Birge, J. R. and Louveaux, F. (1997). Introduction to Stochastic Programming. Springer Series in Operations Research and Financial Engineering. Springer, corrected edition.

Blake, D., Wright, D., and Zhang, Y. (2013). Target-driven investing: Optimal investment strategies in defined contribution pension plans under loss aversion. Journal of Economic Dynamics and Control, 37(1):195-209.

Bruhn, K. (2013). Consumption, investment and life insurance under different tax regimes. Annals of Actuarial Science, 7(2):210-235.

Bruhn, K. and Steffensen, M. (2011). Household consumption, investment and life insurance. Insurance: Mathematics and Economics, 48(3):315-325.

Byung, H. L. and Yong, H. S. (2011). Optimal investment, consumption and retirement decision with disutility and borrowing constraints. Quantitative Finance, 11(10):1581-1592.

Cai, J. and Ge, C. (2012). Multi-objective private wealth allocation without subportfolios. Economic Modelling, 29(3):900-907.

Cairns, A. J. G., Blake, D., and Dowd, K. (2006). Stochastic lifestyling: optimal dynamic asset allocation for defined contribution pension plans. Journal of Economic Dynamics and Control, 30(5):843-877.

Carino, D. R., Myers, D. H., and Ziemba, W. T. (1998). Concepts, technical issues, and uses of the russell-yasuda kasai financial planning model. Operations research, 46(4):450-462.

Chen, Z. and Xu, D. (2013). Knowledge-based scenario tree generation methods and application in multiperiod portfolio selection problem. Applied Stochastic Models in Business and Industry. Article in Press. 
Consigli, G., Iaquinta, G., Moriggia, V., Tria, M. D., and Musitelli, D. (2012). Retirement planning in individual asset-liability management. IMA Journal Management Mathematics, 23(4):365-396.

Danmarks Statistik (2010). Personindkomster. Accessed January 20, 2013, from http://www.dst. $\mathrm{dk} / \mathrm{da} /$ Statistik/emner/indkomster/personindkomster.aspx.

Dempster, M. A. H., Germano, M., Medova, E. A., and Villaverde, M. (2003). Global asset liability management. British Actuarial Journal, 9:137-216.

Ferstl, R. and Weissensteiner, A. (2010). Cash management using multi-stage stochastic programming. Quantitative Finance, 10(2):209-219.

Ferstl, R. and Weissensteiner, A. (2011). Asset-liability management under time-varying investment opportunities. Journal of Banking and Finance, 35(1):182-192.

Finanstilsynet (2010). Benchmark for levetidsforudsaetninger. Accessed January 20, 2012, from http://www.finanstilsynet.dk/levetider.

Gerrard, R., Haberman, S., and Vigna, E. (2004). Optimal investment choices post-retirement in a defined contribution pension scheme. Insurance: Mathematics and Economics, 35(2):321-342.

Gerrard, R., Haberman, S., and Vigna, E. (2006). The management of decumulation risks in a defined contribution pension scheme. North American Actuarial Journal, 10(1):84-110.

Gerrard, R., Højgaard, B., and Vigna, E. (2012). Choosing the optimal annuitization time postretirement. Quantitative Finance, 12(7):1143-1159.

Geyer, A., Hanke, M., and Weissensteiner, A. (2009a). Life-cycle asset allocation and consumption using stochastic linear programming. The Journal of Computational Finance, 12(4):29-50.

Geyer, A., Hanke, M., and Weissensteiner, A. (2009b). A stochastic programming approach for multi-period portfolio optimization. Computational Management Science, 6(2):187-208.

Geyer, A., Hanke, M., and Weissensteiner, A. (2014). No-arbitrage bounds for financial scenarios. European Journal of Operational Research, 236(2):657-663.

Grinold, R. (1977). Finite horizon approximations of infinite horizon linear programs. Mathematical Programming, 12(1):1-17.

Grinold, R. C. (1983). Model building techniques for the correction of end effects in multistage convex programs. Operations Research, 31(3):407-431.

Gülpınar, N., Rustem, B., and Settergren, R. (2004). Simulation and optimization approaches to scenario tree generation. Journal of Economic Dynamics and Control, 28(7):1291-1315.

He, L. and Liang, Z. (2013). Optimal dynamic asset allocation strategy for ELA scheme of DC pension plan during the distribution phase. Insurance: Mathematics and Economics, 52(2):404-410.

Heitsch, H. and Römisch, W. (2009a). Scenario tree modeling for multistage stochastic programs. Mathematical Programming, 118(2):371-406. 
Heitsch, H. and Römisch, W. (2009b). Scenario tree reduction for multistage stochastic programs. Computational Management Science, 6(2):117-133.

Horneff, W. J., Maurer, R. H., and Stamos, M. Z. (2008). Life-cycle asset allocation with annuity markets. Journal of Economic Dynamics and Control, 32(11):3590-3612.

Høyland, K., Kaut, M., and Wallace, S. W. (2003). A heuristic for moment-matching scenario generation. Computational Optimization and Applications, 24(2-3):169-185.

Høyland, K. and Wallace, S. W. (2001). Generating scenario trees for multistage decision problems. Management Science, 48(11):1512-1516.

Ji, X., Zhu, S., Wang, S., and Zhang, S. (2005). A stochastic linear goal programming approach to multistage portfolio management based on scenario generation via linear programming. IIE Transactions (Institute of Industrial Engineers), 37(10):957-969.

Kaut, M. and Wallace, S. W. (2005). Evaluation of scenario generation methods for stochastic programming. Pacific Journal of Optimization, 3(2):257-272.

Kim, W. C., Mulvey, J. M., Simsek, K. D., and Kim, M. J. (2012). Stochastic Programming. Applications in Finance, Energy, Planning and Logistics, chapter Papers in Finance: Longevity risk management for individual investors. World Scientific Series in Finance: Volume 4.

Klaasen, P. (2002). Comment on: "Generating scenario trees for multistage decision problems". Management Science, 47(2):295-307.

Konicz, A. and Mulvey, J. M. (2013). Applying a stochastic financial system for an individual: Immediate or deferred life annuities? The Journal of Retirement, 1(2):46-60.

Kontogiorgis, S. (2000). Practical piecewise-linear approximation for monotropic optimization. INFORMS Journal on Computing, 12(4):324-340.

Kouwenberg, R. (2001). Scenario generation and stochastic programming models for asset liability management. European Journal of Operational Research, 134(2):279-292.

Kraft, H. and Steffensen, M. (2008). Optimal consumption and insurance: a continuous-time markov chain approach. ASTIN Bulletin, 28(1):231-257.

Merton, R. C. (1969). Lifetime portfolio selection under uncertainty: the continuous-time case. The Review of Economics and Statistics, 51(3):247-257.

Merton, R. C. (1971). Optimum consumption and portfolio rules in a continuous-time model. Journal of Economic Theory, 3(4):373-413.

Milevsky and Young (2007). Annuitization and asset allocation. Journal of Economic Dynamics and Control, 31(9):3138-3177.

Mulvey, J. M., Simsek, K. D., and Zhang, Z. (2006). Improving investment performance for pension plans. Journal of Asset Management, 7(2):93-108.

Mulvey, J. M., Ural, C., and Zhang, Z. (2007). Improving performance for long-term investors: wide diversification, leverage, and overlay strategies. Quantitative Finance, 7(2):175-187. 
Munk, C. and Sørensen, C. (2004). Optimal consumption and investment strategies with stochastic interest rates. Journal of Banking and Finance, 28(8):1987-2013.

Rasmussen, K. M. (2011). Finding optimal breakpoints when linearizing a power utility function. Retrieved January 20, 2013, from http://www.gams.com/modlib/libhtml/cclinpts.htm.

Richard, S. F. (1975). Optimal consumption, portfolio and life insurance rules for an uncertain lived individual in a continuous time model. Journal of Financial Economics, 2(2):187-203.

Ruszczyński, A. and Shapiro, A. (2003). Stochastic Programming Models, volume Volume 10 of Handbooks in Operations Research and Management Science, pages 1-64. Elsevier.

Xu, D., Chen, Z., and Yang, L. (2012). Scenario tree generation approaches using K-means and LP moment matching methods. Journal of Computational and Applied Mathematics, 236(17):45614579 .

Zenios, S. (2008). Practical Financial Optimization: Decision Making for Financial Engineers. Wiley.

Ziemba, W. T. and Mulvey, J. M. (1998). Worldwide Asset and Liability Modeling. Publications of the Newton Institute. Cambridge University Press. 


\begin{tabular}{|c|c|c|c|c|c|c|}
\hline control & model & $t_{0}$ & $t_{1}$ & $t_{2}$ & $t_{3}$ & $T_{M S P}-1$ \\
\hline \multirow{9}{*}{$X_{t}^{*}$} & expl. & 60.0 & 72.9 & 86.0 & 99.2 & 112.6 \\
\hline & orig. constraints & 60.0 & 72.8 & 85.8 & 99.0 & 112.4 \\
\hline & & $(0.0)$ & $(0.0)$ & $(0.1)$ & $(0.1)$ & $(0.1)$ \\
\hline & $\pi_{t} \leq 1$ and $I_{t} \geq 0$ & 60.0 & 71.0 & 82.6 & 94.9 & 107.7 \\
\hline & & $(0.0)$ & $(0.0)$ & $(0.0)$ & $(0.0)$ & $(0.0)$ \\
\hline & $t c^{i}=0.5 \%$ & 60.0 & 71.2 & 83.8 & 96.8 & 110.2 \\
\hline & & $(0.0)$ & $(0.0)$ & $(0.0)$ & $(0.1)$ & $(0.1)$ \\
\hline & $\tau^{i}=20 \%$ & 60.0 & 68.6 & 77.5 & 86.6 & 95.9 \\
\hline & & $(0.0)$ & $(0.2)$ & $(0.3)$ & $(0.5)$ & $(0.7)$ \\
\hline \multirow{9}{*}{$\pi_{t}^{*}$} & expl. & 1.80 & 1.50 & 1.27 & 1.10 & 0.97 \\
\hline & orig. constraints & 1.78 & 1.48 & 1.26 & 1.09 & 0.96 \\
\hline & & $(0.01)$ & $(0.00)$ & $(0.00)$ & $(0.00)$ & $(0.00)$ \\
\hline & $\pi_{t} \leq 1$ and $I_{t} \geq 0$ & 1.00 & 1.00 & 1.00 & 0.95 & 0.89 \\
\hline & & $(0.00)$ & $(0.00)$ & $(0.00)$ & $(0.00)$ & $(0.00)$ \\
\hline & $t c^{i}=0.5 \%$ & 1.43 & 1.37 & 1.24 & 1.11 & 1.04 \\
\hline & & $(0.01)$ & $(0.01)$ & $(0.01)$ & $(0.00)$ & $(0.00)$ \\
\hline & $\tau^{i}=20 \%$ & 0.80 & 0.69 & 0.60 & 0.53 & 0.47 \\
\hline & & $(0.08)$ & $(0.07)$ & $(0.06)$ & $(0.05)$ & $(0.05)$ \\
\hline \multirow{9}{*}{$\pi_{t}^{* 1}$} & expl. & 0.60 & 0.50 & 0.42 & 0.37 & 0.32 \\
\hline & orig. constraints & 0.62 & 0.52 & 0.44 & 0.38 & 0.34 \\
\hline & & $(0.01)$ & $(0.01)$ & $(0.01)$ & $(0.01)$ & $(0.01)$ \\
\hline & $\pi_{t} \leq 1$ and $I_{t} \geq 0$ & 0.15 & 0.21 & 0.27 & 0.29 & 0.29 \\
\hline & & $(0.01)$ & $(0.01)$ & $(0.01)$ & $(0.01)$ & $(0.01)$ \\
\hline & $t c^{i}=0.5 \%$ & 0.46 & 0.44 & 0.41 & 0.38 & 0.36 \\
\hline & & $(0.01)$ & $(0.01)$ & $(0.01)$ & $(0.01)$ & $(0.01)$ \\
\hline & $\tau^{i}=20 \%$ & 0.04 & 0.04 & 0.03 & 0.03 & 0.03 \\
\hline & & $(0.05)$ & $(0.05)$ & $(0.04)$ & $(0.04)$ & $(0.03)$ \\
\hline \multirow{9}{*}{$c_{t}^{*}$} & expl. & 20.8 & 20.8 & 20.9 & 20.9 & 20.9 \\
\hline & orig. constraints & 20.8 & 20.8 & 20.9 & 20.9 & 20.9 \\
\hline & & $(0.0)$ & $(0.0)$ & $(0.0)$ & $(0.0)$ & $(0.0)$ \\
\hline & $\pi_{t} \leq 1$ and $I_{t} \geq 0$ & 20.6 & 20.6 & 20.6 & 20.7 & 20.7 \\
\hline & & $(0.0)$ & $(0.0)$ & $(0.0)$ & $(0.0)$ & $(0.0)$ \\
\hline & $t c^{i}=0.5 \%$ & 20.7 & 20.7 & 20.8 & 20.8 & 20.8 \\
\hline & & $(0.0)$ & $(0.0)$ & $(0.0)$ & $(0.0)$ & $(0.0)$ \\
\hline & $\tau^{i}=20 \%$ & 20.5 & 20.4 & 20.3 & 20.2 & 20.1 \\
\hline & & $(0.0)$ & $(0.0)$ & $(0.0)$ & $(0.0)$ & $(0.0)$ \\
\hline \multirow{9}{*}{$I_{t}^{*}$} & expl. & 9.5 & -3.2 & -16.2 & -29.3 & -42.6 \\
\hline & orig. constraints & 9.5 & -3.2 & -16.1 & -29.2 & -42.4 \\
\hline & & $(0.0)$ & $(0.0)$ & $(0.1)$ & $(0.1)$ & $(0.1)$ \\
\hline & $\pi_{t} \leq 1$ and $I_{t} \geq 0$ & 8.8 & 5.0 & 3.0 & 1.7 & 0.9 \\
\hline & & $(0.0)$ & $(0.4)$ & $(0.1)$ & $(0.1)$ & $(0.0)$ \\
\hline & $t c^{i}=0.5 \%$ & 9.3 & -1.9 & -14.4 & -27.3 & -40.5 \\
\hline & & $(0.0)$ & $(0.0)$ & $(0.0)$ & $(0.1)$ & $(0.1)$ \\
\hline & $\tau^{i}=20 \%$ & 8.6 & -0.4 & -9.6 & -18.9 & -28.6 \\
\hline & & $(0.0)$ & $(0.1)$ & $(0.3)$ & $(0.4)$ & $(0.5)$ \\
\hline
\end{tabular}

Table 1: Problem (A). Explicit solution and MSP solution with various modifications for the expected value of the savings, optimal consumption and sum insured (in 1000 EUR) and optimal investment in both risky assets and in the first risky asset $\left(\pi_{t}^{*}=\pi_{t}^{* 1}+\pi_{t}^{* 2}\right)$. The numbers are presented in terms of means and standard errors (in parenthesis). 


\begin{tabular}{|c|c|c|c|c|c|c|}
\hline control & model & $t_{0}$ & $t_{1}$ & $t_{2}$ & $t_{3}$ & $T_{M S P}-1$ \\
\hline \multicolumn{7}{|c|}{ before retirement } \\
\hline \multirow{3}{*}{$X_{t}^{*}$} & expl. & 75.0 & 82.2 & 89.5 & 97.1 & 105.0 \\
\hline & orig. constraints & 75.0 & 82.2 & 89.6 & 97.3 & 105.1 \\
\hline & & $(0.0)$ & $(0.0)$ & $(0.0)$ & $(0.0)$ & $(0.0)$ \\
\hline \multirow{3}{*}{$\pi_{t}^{*}$} & expl. & 0.45 & 0.42 & 0.40 & 0.38 & 0.37 \\
\hline & orig. constraints & 0.44 & 0.42 & 0.40 & 0.38 & 0.36 \\
\hline & & $(0.00)$ & $(0.00)$ & $(0.00)$ & $(0.00)$ & $(0.00)$ \\
\hline \multirow{3}{*}{$\pi_{t}^{* 1}$} & expl. & 0.15 & 0.14 & 0.13 & 0.13 & 0.12 \\
\hline & orig. constraints & 0.16 & 0.15 & 0.14 & 0.13 & 0.13 \\
\hline & & $(0.00)$ & $(0.00)$ & $(0.00)$ & $(0.00)$ & $(0.00)$ \\
\hline \multicolumn{7}{|c|}{ after retirement } \\
\hline \multirow{9}{*}{$X_{t}^{*}$} & expl. & 225.0 & 217.0 & 209.0 & 201.0 & 193.0 \\
\hline & orig. constraints & 225.0 & 216.7 & 208.4 & 200.1 & 191.8 \\
\hline & & $(0.0)$ & $(0.0)$ & $(0.0)$ & $(0.0)$ & $(0.0)$ \\
\hline & $\pi_{t}^{1} \geq 15 \%$ & 225.0 & 216.9 & 208.7 & 200.5 & 192.3 \\
\hline & & $(0.0)$ & $(0.0)$ & $(0.0)$ & $(0.0)$ & $(0.1)$ \\
\hline & $t c^{i}=0.5 \%$ & 225.0 & 215.9 & 207.6 & 199.3 & 190.9 \\
\hline & & $(0.0)$ & $(0.0)$ & $(0.0)$ & $(0.0)$ & $(0.0)$ \\
\hline & $\tau^{i}=20 \%$ & 225.0 & 214.5 & 204.0 & 193.7 & 183.5 \\
\hline & & $(0.0)$ & $(0.1)$ & $(0.1)$ & $(0.2)$ & $(0.3)$ \\
\hline \multirow{9}{*}{$\pi_{t}^{*}$} & expl. & 0.25 & 0.25 & 0.25 & 0.25 & 0.25 \\
\hline & orig. constraints & 0.25 & 0.25 & 0.25 & 0.25 & 0.25 \\
\hline & & $(0.00)$ & $(0.00)$ & $(0.00)$ & $(0.00)$ & $(0.00)$ \\
\hline & $\pi_{t}^{1} \geq 15 \%$ & 0.29 & 0.29 & 0.29 & 0.29 & 0.29 \\
\hline & & $(0.00)$ & $(0.00)$ & $(0.00)$ & $(0.00)$ & $(0.00)$ \\
\hline & $t c^{i}=0.5 \%$ & 0.26 & 0.25 & 0.25 & 0.25 & 0.24 \\
\hline & & $(0.00)$ & $(0.00)$ & $(0.00)$ & $(0.00)$ & $(0.00)$ \\
\hline & $\tau^{i}=20 \%$ & 0.11 & 0.11 & 0.11 & 0.11 & 0.11 \\
\hline & & $(0.01)$ & $(0.01)$ & $(0.01)$ & $(0.01)$ & $(0.01)$ \\
\hline \multirow{9}{*}{$\pi_{t}^{* 1}$} & expl. & 0.08 & 0.08 & 0.08 & 0.08 & 0.08 \\
\hline & orig. constraints & 0.09 & 0.09 & 0.09 & 0.09 & 0.09 \\
\hline & & $(0.00)$ & $(0.00)$ & $(0.00)$ & $(0.00)$ & $(0.00)$ \\
\hline & $\pi_{t}^{1} \geq 15 \%$ & 0.15 & 0.15 & 0.15 & 0.15 & 0.15 \\
\hline & & $(0.00)$ & $(0.00)$ & $(0.00)$ & $(0.00)$ & $(0.00)$ \\
\hline & $t c^{i}=0.5 \%$ & 0.09 & 0.09 & 0.09 & 0.09 & 0.09 \\
\hline & & $(0.00)$ & $(0.00)$ & $(0.00)$ & $(0.00)$ & $(0.00)$ \\
\hline & $\tau^{i}=20 \%$ & 0.01 & 0.01 & 0.01 & 0.01 & 0.01 \\
\hline & & $(0.01)$ & $(0.01)$ & $(0.01)$ & $(0.01)$ & $(0.01)$ \\
\hline \multirow{9}{*}{$c_{t}^{*}$} & expl. & 17.8 & 17.9 & 17.9 & 17.9 & 18.0 \\
\hline & orig. constraints & 17.8 & 17.8 & 17.8 & 17.9 & 17.9 \\
\hline & & $(0.0)$ & (0.0) & $(0.0)$ & $(0.0)$ & $(0.0)$ \\
\hline & $\pi_{t}^{1} \geq 15 \%$ & 17.8 & 17.8 & 17.9 & 17.9 & 18.0 \\
\hline & & $(0.0)$ & $(0.0)$ & $(0.0)$ & $(0.0)$ & $(0.0)$ \\
\hline & $t c^{i}=0.5 \%$ & 17.7 & 17.7 & 17.7 & 17.8 & 17.8 \\
\hline & & $(0.0)$ & $(0.0)$ & $(0.0)$ & $(0.0)$ & $(0.0)$ \\
\hline & $\tau^{i}=20 \%$ & 17.3 & 17.3 & 17.2 & 17.1 & 17.0 \\
\hline & & $(0.0)$ & $(0.0)$ & $(0.0)$ & $(0.0)$ & $(0.0)$ \\
\hline
\end{tabular}

Table 2: Problem (B) before and after retirement. Explicit solution and MSP solution with various modifications for the expected value of the savings and optimal benefits (in 1000 EUR), and optimal investment in both risky assets and in the first risky asset $\left(\pi_{t}^{*}=\pi_{t}^{* 1}+\pi_{t}^{* 2}\right)$. The numbers are presented in terms of means and standard errors (in parenthesis). 